\title{
Somatic mutation rates scale with lifespan across mammals
}

Authors

$5{\text { Alex } \text { Cagan }^{1+*} \text {, Adrian Baez-Ortega }}^{1 *}$, Natalia Brzozowska ${ }^{1}$, Federico Abascal ${ }^{1}$, Tim H. H.

6 Coorens $^{1}$, Mathijs A. Sanders ${ }^{1,2}$, Andrew R. J. Lawson ${ }^{1}$, Luke M. R. Harvey ${ }^{1}$, Shriram Bhosle ${ }^{1}$,

7 David Jones $^{1}$, Raul E. Alcantara ${ }^{1}$, Timothy M. Butler ${ }^{1}$, Yvette Hooks ${ }^{1}$, Kirsty Roberts ${ }^{1}$, Elizabeth

8 Anderson 1 , Sharna Lunn ${ }^{1}$, Edmund Flach ${ }^{3}$, Simon Spiro ${ }^{3}$, Inez Januszczak ${ }^{3,4}$, Ethan

9 Wrigglesworth ${ }^{3}$, Hannah Jenkins ${ }^{3}$, Tilly Dallas ${ }^{3}$, Nic Masters ${ }^{3}$, Matthew W. Perkins ${ }^{5}$, Robert

10 Deaville $^{5}$, Megan Druce ${ }^{6,7}$, Ruzhica Bogeska ${ }^{6,7}$, Michael D. Milsom ${ }^{6,7}$, Björn Neumann ${ }^{8,9}$, Frank

11 Gorman $^{10}$, Fernando Constantino-Casas ${ }^{10}$, Laura Peachey ${ }^{10,11}$, Diana Bochynska ${ }^{10,12}$, Ewan St.

12 John Smith $^{13}$, Moritz Gerstung ${ }^{14}$, Peter J. Campbell ${ }^{1}$, Elizabeth P. Murchison ${ }^{10}$, Michael R.

13 Stratton ${ }^{1}$, Iñigo Martincorena ${ }^{1+}$

$15 *$ These authors contributed equally

$16+$ Correspondence to: ac36@sanger.ac.uk (A.C.) and im3@sanger.ac.uk (I.M.)

Author information

19 (1) Cancer, Ageing and Somatic Mutation (CASM), Wellcome Sanger Institute, Hinxton, CB10

20 1SA, UK

21 (2) Department of Hematology, Erasmus MC Cancer Institute, 3015 CN Rotterdam, Netherlands

22 (3) Wildlife Health Services, Zoological Society of London, Regent's Park, London, NW1 4RY, 23 UK

24 (4) The Natural History Museum, Cromwell Road, London, SW7 5BD, UK

25 (5) Institute of Zoology, Zoological Society of London, Regent's Park, London, NW1 4RY, UK

26 (6) Division of Experimental Hematology, German Cancer Research Center (DKFZ), 69120

27 Heidelberg, Germany

28 (7) Heidelberg Institute for Stem Cell Technology and Experimental Medicine GmbH (HI-

29 STEM), 69120 Heidelberg, Germany

30 (8) Wellcome Trust-Medical Research Council Cambridge Stem Cell Institute, University of

31 Cambridge, Cambridge, CB2 0AW, UK

32 (9) Department of Clinical Neurosciences, University of Cambridge, Cambridge, CB2 0QQ, UK 
33 (10) Department of Veterinary Medicine, University of Cambridge, Cambridge, CB3 0ES, UK

34 (11) Bristol Veterinary School, Faculty of Health Sciences, University of Bristol, Langford, BS40

35 5DU, UK

36 (12) (12) Pathology Department, Faculty of Veterinary Medicine, Universitatea de Stiinte

37 Agricole si Medicina Veterinara, Cluj-Napoca 400372, Romania

38 (13) Department of Pharmacology, University of Cambridge, Cambridge CB2 1PD, UK

39 (14) European Molecular Biology Laboratory, European Bioinformatics Institute (EMBL-EBI),

40 Hinxton, CB10 1SD, UK

\section{Abstract}

The rates and patterns of somatic mutation in normal tissues are largely unknown outside of humans. Comparative analyses can shed light on the diversity of mutagenesis across species and on long-standing hypotheses regarding the evolution of somatic mutation rates and their role in cancer and ageing. Here, we used whole-genome sequencing of 208 intestinal crypts from 56 individuals to study the landscape of somatic mutation across 16 mammalian species. We found somatic mutagenesis to be dominated by seemingly endogenous mutational processes in all species, including 5-methylcytosine deamination and oxidative damage. With some differences, mutational signatures in other species resembled those described in humans, although the relative contribution of each signature varied across species. Remarkably, the somatic mutation rate per year varied greatly across species and exhibited a strong inverse relationship with species lifespan, with no other life-history trait studied displaying a comparable association. Despite widely different life histories among the species surveyed, including $\sim 30$-fold variation in lifespan and $\sim 40,000$-fold variation in body mass, the somatic mutation burden at the end of lifespan varied only by a factor of $\sim 3$. These data unveil common mutational processes across mammals and suggest that somatic mutation rates are evolutionarily constrained and may be a contributing factor in ageing.

\section{Introduction}


64 Somatic mutations accumulate in healthy cells throughout life. They underpin cancer

65 development ${ }^{1,2}$ and, for decades, have been speculated to contribute to ageing ${ }^{3-5}$. Directly studying

66 somatic mutations in normal tissues has been challenging due to the difficulty of detecting

67 mutations present in single cells or small clones in a tissue. Only recent technological

68 developments, such as in vitro expansion of single cells into colonies ${ }^{6,7}$, microdissection of

69 histological units ${ }^{8,9}$ or single-cell or single-molecule sequencing ${ }^{10-12}$, are beginning to enable the

70 study of somatic mutation in normal tissues.

72 Over the last few years, studies in humans have started to provide a detailed understanding of 73 somatic mutation rates and the contribution of endogenous and exogenous mutational processes 74 across normal tissues ${ }^{6-8,13,14}$. These studies are also revealing how, as we age, some human tissues 75 are colonised by mutant cells carrying cancer-driving mutations, and how this clonal composition 76 changes with age and disease. With the exception of some initial studies, far less is known about

77 somatic mutation in other species ${ }^{15-20}$. Yet, comparative analyses of somatic mutagenesis would 78 shed light on the diversity of mutagenic processes across species, and on long-standing questions regarding the evolution of somatic mutation rates and their role in cancer and ageing.

A decades-long hypothesis on the evolution of somatic mutation rates pertains to the relationship between body mass and cancer risk. Some models predict that the risk of cancer should increase proportionally to the number of cells at risk of transformation. However, there appears to be no correlation between body mass and cancer risk across species ${ }^{21-23}$. This observation, known as Peto's paradox, suggests that the evolution of larger body sizes likely requires the evolution of stronger cancer suppression mechanisms ${ }^{23,24}$. Whether evolutionary reduction of cancer risk across species is partly achieved by a reduction of somatic mutation rates remains unknown ${ }^{25}$.

A second long-standing hypothesis on the evolution of somatic mutation rates relates to the proposed role of somatic mutations in ageing. Multiple forms of molecular damage, including somatic mutations, telomere attrition, epigenetic drift or loss of proteostasis, have been proposed 92 to contribute to ageing, but their causal roles and relative contributions remain debated ${ }^{26,27}$.

93 Evolutionary theory predicts that species will evolve protection or repair mechanisms against life-

94 threatening damage to minimise death from intrinsic causes, but that selection is too weak to delay 95 ageing far beyond the typical life expectancy of an organism in the wild ${ }^{28-32}$ (Supplementary Note 
1). If somatic mutations contribute to ageing, theory predicts that somatic mutation rates may inversely correlate with lifespan across species ${ }^{30,33}$. This long-standing prediction has remained largely untested due to the difficulty of measuring somatic mutation rates across species.

\section{Detection of somatic mutations across species}

The study of somatic mutations with standard whole-genome sequencing requires isolating clonal groups of cells recently derived from a single cell ${ }^{6-8}$. To study somatic mutations across a diverse set of mammals, we isolated 208 individual intestinal crypts from 56 individuals across 16 species with a wide range of lifespans and body sizes: black-and-white colobus monkey, cat, cow, dog, ferret, giraffe, harbour porpoise, horse, human, lion, mouse, naked mole-rat, rabbit, rat, ring-tailed lemur, and tiger (Supplementary Table 1). We chose intestinal crypts for several reasons. First, they are histologically identifiable units that line the epithelium of the colon and small intestine and are amenable to laser microdissection. Second, human studies have confirmed that individual crypts become clonally derived from a single stem cell and show a linear accumulation of mutations with age, enabling the estimation of somatic mutation rates through genome sequencing of single crypts $^{8}$. Third, in most human crypts, the majority of somatic mutations are caused by endogenous mutational processes common to other tissues, rather than by environmental mutagens ${ }^{8,12}$.

115 A colon sample was collected from each individual, with the exception of a ferret from which only 116 a small intestine sample was available. This sample was included because results in humans have 117 shown the mutation rate of colorectal and small intestine epithelium to be similar, ${ }^{7}, 14$ (Extended 118 Data Figure 1). We then used laser microdissection on histological sections to isolate individual 119 crypts for whole-genome sequencing with a low-input library preparation method ${ }^{34}$ (Fig. 1a, 120 Extended Data Figure 2, Supplementary Table 2), with the exception of human crypts, for which 121 sequencing data were obtained from a previous study ${ }^{8}$. A bioinformatic pipeline was developed to 122 call somatic mutations robustly in all these species despite variable quality of their genome 123 assemblies (Methods). The distribution of variant allele fractions (VAFs) of the mutations detected 124 in each crypt confirmed that crypts are clonal units in all species, enabling the study of somatic 125 mutation rates and signatures (Extended Data Figure 3). 
127 We found substantial variation in the number of somatic single-base substitutions (SBS) across

128 species and across individuals within each species (Fig. 1b). For five species with samples from

129 multiple individuals, linear regression confirmed a clear accumulation of somatic mutations with

130 age (Fig. 1c, Extended Data Figure 4, Supplementary Table 3). All linear regressions were also

131 consistent with a non-significant intercept. This resembles observations in humans ${ }^{14}$ and suggests

132 that the time required for a single stem cell to drift to fixation within a crypt is a small fraction of

133 the lifespan of a species. This facilitates the estimation of somatic mutation rates across species by

134 dividing the number of mutations in a crypt by the age of the individual (Supplementary Table

135 4). The number of somatic insertions and deletions (indels) was consistently lower than that of SBS

136 in all crypts (Fig. 1b), in agreement with previous findings in humans ${ }^{8}$.

\section{Similar mutational signatures across mammals}

140 Somatic mutations can be caused by different mutational processes, including multiple forms of

141 DNA damage and repair. Different processes cause characteristic frequencies of base substitution

142 types and indels at different sequence contexts, often referred to as mutational signatures, which

143 can be inferred from mutation data ${ }^{35,36}$. Across species, the mutational spectra showed clear

144 similarities, with a dominance of cytosine-to-thymine $(\mathrm{C}>\mathrm{T})$ substitutions at $\mathrm{CpG}$ sites, as observed

145 in human colon, but with considerable variation in the frequency of other substitution types (Fig.

146 2a). To quantify the contribution of different mutational processes to the observed spectra, we

147 applied mutational signature decomposition ${ }^{8,35}$. We used a Bayesian model to infer mutational

148 signatures de novo, while accounting for differences in genome sequence composition across

149 species, and using the COSMIC human signature SBS1 (C>T substitutions at CpG sites) as a fixed

150 prior to ensure its complete deconvolution ${ }^{37}$ (Methods). This approach identified two signatures

151 beyond SBS1, labelled SBSB and SBSC, which resemble COSMIC human signatures SBS5 and

152 SBS18, respectively (cosine similarities 0.93 and 0.91) (Fig. 2b).

154 This analysis suggests that the same three signatures that dominate somatic mutagenesis in the

155 human colon are dominant in other mammals: SBS1, which is believed to result from the

156 spontaneous deamination of 5-methylcytosine ${ }^{8,38}$; SBSB/5, a common signature across human

157 tissues that may result from endogenous damage and repair ${ }^{12,39}$; and $\mathrm{SBSC} / 18$, dominated by $\mathrm{C}>\mathrm{A}$

158 substitutions and attributed to oxidative DNA damage ${ }^{36}$. Signature SBSC contains a minor 
component of T $>$ A substitutions (resembling COSMIC SBS34), which appear to be the result of

160 DNA polymerase slippage at the boundaries between adjacent adenine and thymine homopolymer

161 tracts but that could also reflect assembly errors at those sites ${ }^{39}$. While all species surveyed shared

162 the three mutational signatures, their contributions varied substantially across species (Fig. 2c).

163 SBSC was particularly prominent in mouse and ferret, and the ratio of SBS1 to SBSB (SBS5)

164 varied from approximately 1.2 in rat or rabbit to 6.4 in tiger. In several species with data from

165 multiple individuals, separate linear regressions for each signature confirmed that mutations from

166 all three signatures accumulate with age (Fig. 2d, Extended Data Figure 5).

168 Although signature deconvolution identified three signatures active across species, we noticed

169 some differences in the mutational profile of signature SBSB among species. To explore this

170 further, we inferred independent versions of SBSB from each species, while accounting for

171 differences in genome sequence composition (Methods). This revealed inter-species variability in

172 the mutational profile of this signature, particularly in the $\mathrm{C}>\mathrm{T}$ component (Extended Data Figure

173 6). Species-specific versions of SBSB showed different similarities to the related human signatures

174 SBS5 and SBS40. For example, SBSB inferred from the human data showed a stronger similarity

175 with the reference SBS5 human signature (cosine similarities with SBS5 and SBS40: 0.93 and

176 0.84), whereas SBSB from rabbit more closely resembled the reference human SBS40 signature

177 (0.87 and 0.91). These observations are consistent with the hypothesis that SBS5 and SBS40 result

178 from a combination of correlated mutational processes, with some variation across human

179 tissues $^{12,39}$ and across species.

181 Analysis of the indel mutational spectra revealed a dominance of the human indel signatures ID1

182 and ID2, characterised by single-nucleotide insertions and deletions at A/T homopolymers, and

183 probably caused by strand slippage during DNA replication ${ }^{36}$ (Extended Data Figure 7). The ratio

184 of insertions (ID1) to deletions (ID2) appears to vary across species, possibly reflecting a

185 differential propensity for slippage of the template and nascent DNA strands ${ }^{36}$. In addition, the

186 indel spectra suggest a potential contribution of signature ID9 (whose aetiology remains unknown)

187 to human, colobus, cow, giraffe and rabbit. Analysis of indels longer than 1 base pair (bp) also

188 suggested the presence of a signature of 4-bp insertions at tetrameric repeats, particularly prevalent

189 in mouse and tiger; a pattern of $\geq 5$-bp insertions at repeats in colobus; and a pattern of $\geq 5$-bp 
190 deletions at repeats prominent in rabbit, which resembles ID8 (a signature possibly caused by

191 double-strand break repair via non-homologous end joining ${ }^{36}$ ) (Extended Data Figure 7).

\section{Other mutational processes and selection}

The apparent lack of additional mutational signatures is noteworthy. A previous study of 445 colorectal crypts from 42 human donors found many crypts to be affected by a novel signature that was later attributed to colibactin, a genotoxin produced by $p k s+$ strains of Escherichia coli ${ }^{8,40,41}$. Analysing the original human data and our non-human data with the same methodology, we found evidence of colibactin mutagenesis in $21 \%$ of human crypts but only uncertain evidence of colibactin in one non-human crypt (0.6\%) (Extended Data Figure 8, Methods). This revealed a significant depletion of colibactin mutagenesis in the non-human crypts studied (Fisher's exact tests, $\left.P=7 \times 10^{-14}\right)$. The apparent difference in colibactin mutagenesis observed between species, or between the cohorts studied, might result from a different prevalence of $p k s+E$. coli strains ${ }^{42}$ or a different expression of colibactin by $p k s+E . c o l i$ across species ${ }^{43}$. Finally, we also searched for evidence of APOBEC signatures (SBS2/13), which have been reported in a small number of human crypts and are believed to be caused by APOBEC DNA-editing cytidine deaminases. We detected APOBEC signatures in $2 \%(n=9)$ of human crypts and only uncertain evidence in one non-human crypt $(P=0.30)$.

Beyond single-base substitutions and indels, crypts from the eight species with chromosome-level

211 genome assemblies were inspected for large-scale copy number changes $(\geq 1$ megabase, Mb)

212 (Methods). Studies in humans have found large-scale copy number changes to be relatively rare

213 in normal tissues, including colorectal epithelium ${ }^{8}$. Consistent with these results, we only identified

214 four large copy number changes across the 162 crypts included in this analysis: two megabase-

215 scale deletions in two crypts from the same cow, the loss of a chromosome $\mathrm{X}$ in a female mouse

216 crypt, and a 52-Mb segment with copy-neutral loss of heterozygosity in a human crypt (Extended

217 Data Figure 9, Methods). These results suggest that large-scale somatic copy number changes in

218 normal tissues are also rare in other mammalian species.

220 Previous analyses in humans have shown that most somatic mutations in colorectal crypts 
mutations and with a low frequency of positively selected cancer-driver mutations ${ }^{8}$. To study somatic selection in our data, we calculated the exome-wide ratio of non-synonymous to synonymous substitution rates $(\mathrm{dN} / \mathrm{dS})$ in each of the 12 species with available genome annotation. To do so and to detect genes under positive selection, while accounting for the effects of trinucleotide sequence context and mutation rate variation across genes, we used the $\mathrm{dNdScv}$ model $^{44}$ (Methods). Although the limited number of coding somatic mutations observed in most species prevented an in-depth analysis of selection, exome-wide $\mathrm{dN} / \mathrm{dS}$ ratios for somatic substitutions were not significantly different from unity in any species, in line with previous findings in humans ${ }^{8}$ (Extended Data Figure 10). Gene-level analysis did not find genes under significant positive selection in any species, although larger studies are likely to identify rare cancer-driver mutations ${ }^{8}$.

\section{Somatic mutation rates and life-history traits}

Whereas similar mutational processes operate across the species surveyed, the mutation rate per genome per year varied widely. Across the 15 species with age information, we found that substitution rates per genome ranged from $47 \mathrm{SBS} /$ year in humans to $796 \mathrm{SBS} /$ year in mice, and indel rates from 2.5 to 158 indels/year, respectively (Fig. 3a, Supplementary Table 4, Methods).

To explore the relationship between somatic mutation rates, lifespan and other life-history traits,

242 we first estimated the lifespan of each species using survival curves. We used a large collection of mortality data from animals in zoos to minimise the impact of extrinsic mortality (Extended Data

244 Figure 11). We defined lifespan as the age at which $80 \%$ of individuals reaching adulthood have died, to reduce the impact of outliers and variable cohort sizes that affect maximum lifespan estimates $^{45,46}$ (Methods). Remarkably, we found a tight anticorrelation between somatic mutation rates per year and lifespan across species (Fig. 3b). A log-log allometric regression yielded a strong

248 linear anticorrelation between mutation rate per year and lifespan (fraction of variance explained, $\mathrm{FVE}=0.85, P=1 \times 10^{-6}$ ), with a slope close to and non-significantly different from -1 . This supports a simple model in which somatic mutation rates per year are inversely proportional to the lifespan

251 of a species (rate $\propto 1 /$ lifespan), such that the number of somatic mutations per cell at the end of the 252 lifespan (the end-of-lifespan burden, ELB) is similar in all species (Fig. 3a-c). 
254 To further study the relationship between somatic mutation rates and life-history variables, we used

255 linear mixed-effects regression models. These models account for the hierarchical structure of the

256 data (with multiple crypts per individual and multiple individuals per species), as well as the

257 heteroscedasticity of somatic mutation rate estimates across species (Methods). Using these

258 models, we estimated that the inverse of lifespan explained $82 \%$ of the inter-species variance in

259 somatic substitution rates (rate $=k$ /lifespan $)\left(P=2.9 \times 10^{-9}\right.$; Fig. 3c), with the slope of this regression

260 (k) representing the mean estimated ELB across species (3206.4 substitutions per genome per crypt,

$26195 \%$ confidence interval 2683.9-3728.9). Strikingly, despite uncertainty in the estimates of both

262 somatic mutation rates and lifespans, and despite the diverse life histories of the species surveyed,

263 including $\sim 30$-fold variation in lifespan and $\sim 40,000$-fold variation in body mass, the estimated

264 mutation load per cell at the end of lifespan varied by only $\sim 3$-fold across species (Table 1).

265 Analogous results were obtained repeating the analysis with protein-coding mutation rate

266 estimates, which may be a better proxy for the functional impact of somatic mutations (85\% of

267 variance explained, ELB: 31 coding substitutions per crypt) (Extended Data Figure 12,

268 Methods).

270 We next explored the association between somatic mutation rates and adult body mass, which is

271 known to be a common confounder in correlations involving lifespan ${ }^{47,48}$. An anticorrelation

272 between somatic mutation rates and body mass may be expected if the modulation of cancer risk

273 across species of vastly different sizes has been a major factor in the evolution of somatic mutation

274 rates. We observed that log-transformed adult body mass was less strongly associated with the

275 somatic substitution rate (allometric regression $F V E=0.21$, Fig. 3d; linear mixed-effect regression

276 FVE=0.44, Fig. 3e). Given that lifespan is correlated with body mass, we performed two tests to

277 assess whether body mass explained any variation in somatic mutation rates not explained by

278 lifespan. First, including both lifespan and log-transformed adult body mass in the regression model

279 suggested that body mass does not explain a significant amount of variance in somatic mutation

280 rates across species after accounting for the effect of lifespan (likelihood ratio tests: $P=0.16$ for

281 body mass on a model with lifespan, $P<10^{-4}$ for lifespan on a model with body mass; Fig. 3f,

282 Methods). Second, partial correlation analyses using allometric regressions further confirmed that

283 the association between somatic mutation rates and lifespan is unlikely to be mediated by the effect

284 of body mass on both variables (lifespan residuals: $P=3.2 \times 10^{-6}, \mathrm{FVE}=0.82$, Fig. 3b; body mass

285 residuals: $P=0.39, \mathrm{FVE}=0.06$, Fig. 3d; Methods). 
287 The fact that the variation in somatic mutation rates across species appears to be dominated by 288 lifespan rather than body size is also apparent when looking at particularly informative species. Giraffe and naked mole-rat, for instance, have similar somatic mutation rates (99 and 93 substitutions/year), in line with their similar lifespans (80th percentiles: 24 and 25 years), despite a $\sim 23,000$-fold difference in adult body mass (Fig. 3d,e). Cows, giraffes and tigers weigh much more than an average human, and yet have somatic mutation rates several fold higher, in line with expectation from their lifespans but not their body mass. Altogether, the weak correlation between body mass and somatic mutation rates after correction for lifespan suggests that the evolution of larger body sizes may have relied on alternative or additional strategies to limit cancer risk, as has

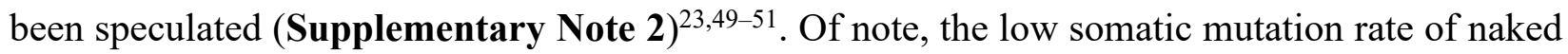
mole-rats, unusual for their body mass but in line with their long lifespan (Fig. 3d,e), might contribute to the exceptionally low cancer incidence rates of this species ${ }^{52,53}$.

We found similar results for other life-history variables that have been proposed to correlate with lifespan, namely basal metabolic rate $(B M R)$ and litter size ${ }^{54,55}$ (Fig. 3f). With the caveat that estimates for these variables vary in quality, they showed weaker correlations with the somatic mutation rate as single predictors, and small non-significant increases in explanatory power when considered together with lifespan (likelihood ratio tests, $P=0.92$ for litter size, $\mathrm{P}=0.083$ for $\log$ $\mathrm{BMR}, P=0.79$ for allometric BMR residuals; Fig. 3f, Methods). We note that the results above are robust to the use of alternative measures of the somatic mutation rate, including the rate per exome or mutations/Mb (Extended Data Figure 12, Methods); alternative estimates of lifespan, including maximum lifespan (Extended Data Figure 13, Methods); alternative regression regression, which accounts for the effect of phylogenetic relationships (Extended Data Figure

311 14a,b, Methods); and bootstrapping analyses at the level of individuals or species (Extended Data

312 Figure 14c, Methods).

\section{Mutational processes and lifespan}

316 To explore whether a single biological process could drive the association between somatic 317 mutation rates and lifespan, we analysed each mutational signature separately. SBS1, SBSB/5 and 
$318 \mathrm{SBSC} / 18$ are believed to result from different forms of DNA damage and are expected to be subject 319 to different DNA repair pathways ${ }^{12,39,56}$. They also appear to differ in their association with the rate

320 of cell division in humans, with SBS1 being more common in fast-proliferating tissues, such as

321 colon and embryonic or foetal tissues, and SBS5 dominating in post-mitotic cells in the absence of

322 cell division ${ }^{7,12,14}$. Overall, we found clear anticorrelations between mutation rates per year and

323 lifespan for the three SBS signatures and for indels, suggesting that a single biological process or

324 DNA repair pathway is unlikely to be responsible for this association (Fig. 4). The total mutation

325 burden also appears to show a closer fit with lifespan than individual mutational processes, as

326 measured by the range of end-of-lifespan-burden (ELB) for each process across species (Fig. 4).

327 This may be expected if the observed anticorrelation were the result of evolutionary pressure on

328 somatic mutation rates.

330 DNA damage and somatic mutations in the mitochondrial genome have also attracted considerable 331 interest in the ageing field ${ }^{57,58}$. Our whole-genome sequencing of individual crypts provided high 332 coverage of the mitochondrial genome, ranging from 2,188 to 29,691-fold. Normalised against the 333 nuclear coverage, these data suggest that colorectal crypts contained on the order of $\sim 100-2,000$ 334 mitochondrial genomes per cell (Extended Data Figure 15). Using a mutation calling algorithm 335 sensitive to low-frequency variants, we found a total of 261 mitochondrial mutations across 199 336 crypts (Methods). The mutational spectra across species appeared broadly consistent with that 337 observed in humans, with a dominance of $C>T$ and $A>G$ substitutions believed to result from 338 mtDNA replication errors rather than DNA damage ${ }^{59}$ (Extended Data Figure 16). While the low 339 number of mitochondrial mutations detected per species precludes a detailed analysis, the estimated 340 number of somatic mutations per copy of mtDNA also appears to show a significant anticorrelation 341 with lifespan. Across species, we obtained an average of 0.23 detectable mutations per copy of the

342 mitochondrial genome by the end of lifespan (Fig. 4, Methods), a considerable burden given the 343 coding-sequence density and functional relevance of the mitochondrial genome.

\section{Discussion}

347 Using whole-genome sequencing of 208 colorectal crypts from 56 individuals, we provide insights 348 into the somatic mutational landscape of 16 mammalian species. Despite their different diets and 349 life histories, we found remarkable similarities in their mutational spectra. Three main mutational 
350 signatures explain the spectra across species, albeit with varying contributions and subtle variations

351 in the profile of signature SBSB. These results suggest that, at least in the colorectal epithelium, a

352 conserved set of mutational processes dominate somatic mutagenesis across mammals.

354 The most striking finding of this study is the inverse scaling of somatic mutation rates with lifespan,

355 a long-standing prediction of the somatic mutation theory of ageing ${ }^{4,30,33}$. Considering evolutionary

356 and mechanistic models of ageing together provides a framework for discussing the possible

357 implications of these results for ageing (Supplementary Note 1). Jointly, these models predict

358 ageing to be multifactorial, with multiple forms of molecular and cellular damage contributing to

359 organismal ageing due to evolutionary limits to selection acting on the rates of these processes.

360 The inverse scaling of somatic mutation rates and lifespan is consistent with somatic mutations

361 contributing to ageing and with somatic mutation rates being evolutionarily constrained, although

362 we discuss alternative explanations below. This interpretation is also supported by studies reporting

363 more efficient DNA repair in longer-lived species ${ }^{60-62}$. Somatic mutations could contribute to

364 ageing in different ways. Traditionally, they have been proposed to contribute to ageing through

365 deleterious effects on cellular fitness ${ }^{4,63}$, but recent findings question this assumption

366 (Supplementary Note 1). Instead, the discovery of widespread clonal expansions in ageing human

367 tissues ${ }^{13,64-66}$ raises the possibility that some somatic mutations contribute to ageing by increasing

368 the fitness of mutant cells at a cost to the organism ${ }^{63,67,68}$. Recent examples include the possible

369 links between clonal haematopoiesis and cardiovascular disease ${ }^{69}$, between mutations in liver

370 disease and insulin resistance ${ }^{70}$, and between driver mutations in cavernomas and brain

371 haemorrhages ${ }^{63,67,68}$. Detailed studies on the extent and impact of somatic mutations and clonal

372 expansions in age-related diseases may help clarify the precise role, if any, of somatic mutations

373 in ageing. Even if clear causal links between somatic mutations and ageing are established, ageing

374 is likely to be multifactorial. Other forms of molecular damage involved in ageing could be

375 expected to display similar anticorrelations with lifespan and, indeed, such anticorrelations have

376 been reported for telomere shortening and protein turnover ${ }^{71,72}$.

378 Alternative non-causal explanations for the observed anticorrelation between somatic mutation

379 rates and lifespan need to be considered. One alternative explanation is that cell division rates scale

380 with lifespan and explain the observed somatic mutation rates. Available estimates of cell division

381 rates, though imperfect and limited to a few species, do not readily support this argument 
382 (Methods). More importantly, studies in humans have shown that cell division rates are not a major 383 determinant of somatic mutation rates across human tissues ${ }^{7,12}$. Another alternative explanation for

384 the observed anticorrelation might be that selection acts to reduce germline mutation rates in 385 species with longer reproductive spans, in turn causing an anticorrelation of somatic mutation rates 386 and lifespan. While selective pressure on germline mutation rates could influence somatic mutation 387 rates, it is unlikely that germline mutation rates determine somatic mutation rates: somatic mutation 388 rates in humans are 10-20 times higher than germline mutation rates, show variability across cell 389 types, and are influenced by additional mutational processes ${ }^{12,14}$. Overall, the strong scaling of 390 somatic mutation rates with lifespan across mammals, despite variable contributions of different 391 mutational processes, suggests that somatic mutation rates themselves have been evolutionarily 392 constrained, possibly through selection on multiple DNA repair pathways. Alternative explanations 393 need to be able to explain the strength of the scaling despite these differences.

Altogether, this study provides an unprecedented description of somatic mutation across mammals, identifying common and variable features and shedding light on long-standing hypotheses. Scaled across the tree of life and across tissues, in species with vastly different physiologies, life histories, genome compositions and mutagenic exposures, similar studies promise to transform our understanding of somatic mutation and its impact on evolution, ageing, and disease.

\section{References}

403 1. Nowell, P. C. The clonal evolution of tumor cell populations. Science 194, 23-28 (1976).

404 2. Stratton, M. R., Campbell, P. J. \& Futreal, P. A. The cancer genome. Nature 458, 719-724 (2009).

405 3. Szilard, L. ON THE NATURE OF THE AGING PROCESS. Proc. Natl. Acad. Sci. U. S. A. 45, 30$406 \quad 45(1959)$.

407 4. Morley, A. A. The somatic mutation theory of ageing. Mutat. Res. 338, 19-23 (1995).

408 5. Vijg, J. \& Dong, X. Pathogenic Mechanisms of Somatic Mutation and Genome Mosaicism in Aging. $409 \quad$ Cell 182, 12-23 (2020).

410 6. Welch, J. S. et al. The origin and evolution of mutations in acute myeloid leukemia. Cell 150, 264$278(2012)$. 
412 7. Blokzijl, F. et al. Tissue-specific mutation accumulation in human adult stem cells during life. Nature

$413 \quad$ 538, 260-264 (2016).

414 8. Lee-Six, H. et al. The landscape of somatic mutation in normal colorectal epithelial cells. Nature

$415 \quad \mathbf{5 7 4}, 532-537(2019)$.

416 9. Moore, L. et al. The mutational landscape of normal human endometrial epithelium. Nature 580,

$417 \quad 640-646(2020)$.

418 10. Lodato, M. A. et al. Aging and neurodegeneration are associated with increased mutations in single

419 human neurons. Science 359, 555-559 (2018).

420 11. Zhang, L. et al. Single-cell whole-genome sequencing reveals the functional landscape of somatic 421 mutations in B lymphocytes across the human lifespan. Proc. Natl. Acad. Sci. U. S. A. 116, 9014 $4229019(2019)$.

423 12. Abascal, F. et al. Somatic mutation landscapes at single-molecule resolution. Nature (2021)

424 doi:10.1038/s41586-021-03477-4.

425 13. Martincorena, I. et al. Tumor evolution. High burden and pervasive positive selection of somatic 426 mutations in normal human skin. Science 348, 880-886 (2015).

427 14. Moore, L. et al. The mutational landscape of human somatic and germline cells. Cold Spring Harbor Laboratory 2020.11.25.398172 (2020) doi:10.1101/2020.11.25.398172.

429 15. Garcia, A. M. et al. Age- and temperature-dependent somatic mutation accumulation in Drosophila $430 \quad$ melanogaster. PLoS Genet. 6, e1000950 (2010).

431 16. Behjati, S. et al. Genome sequencing of normal cells reveals developmental lineages and mutational 432 processes. Nature 513, 422-425 (2014).

433 17. Milholland, B. et al. Differences between germline and somatic mutation rates in humans and mice. $434 \quad$ Nat. Commun. 8, 15183 (2017).

435 18. Schmid-Siegert, E. et al. Low number of fixed somatic mutations in a long-lived oak tree. Nat Plants $436 \quad 3,926-929(2017)$.

437 19. Orr, A. J. et al. A phylogenomic approach reveals a low somatic mutation rate in a long-lived plant. 
Proc. Biol. Sci. 287, 20192364 (2020).

439 20. López, E. H. \& Palumbi, S. R. Somatic Mutations and Genome Stability Maintenance in Clonal $440 \quad$ Coral Colonies. Mol. Biol. Evol. 37, 828-838 (2020).

441 21. Peto, R., Roe, F. J., Lee, P. N., Levy, L. \& Clack, J. Cancer and ageing in mice and men. Br. J. Cancer 32, 411-426 (1975).

22. Caulin, A. F. \& Maley, C. C. Peto's Paradox: evolution's prescription for cancer prevention. Trends Ecol. Evol. 26, 175-182 (2011).

23. Tollis, M., Boddy, A. M. \& Maley, C. C. Peto's Paradox: how has evolution solved the problem of cancer prevention? BMC Biol. 15, 60 (2017).

24. Peto, R. Epidemiology, multistage models, and short-term mutagenicity tests. Int. J. Epidemiol. 45, 621-637 (2016).

25. Caulin, A. F., Graham, T. A., Wang, L.-S. \& Maley, C. C. Solutions to Peto's paradox revealed by mathematical modelling and cross-species cancer gene analysis. Philos. Trans. R. Soc. Lond. B Biol. Sci. 370, (2015).

26. López-Otín, C., Blasco, M. A., Partridge, L., Serrano, M. \& Kroemer, G. The hallmarks of aging. Cell 153, 1194-1217 (2013).

27. Schumacher, B., Pothof, J., Vijg, J. \& Hoeijmakers, J. H. J. The central role of DNA damage in the ageing process. Nature 592, 695-703 (2021).

28. Medawar, P. B. UNSOLVED problem of biology. Med. J. Aust. 1, 854-855 (1953).

29. Williams, G. C. Pleiotropy, Natural Selection, and the Evolution of Senescence. Evolution 11, 398411 (1957).

30. Kirkwood, T. B. \& Holliday, R. The evolution of ageing and longevity. Proc. R. Soc. Lond. B Biol. Sci. 205, 531-546 (1979).

461 31. Partridge, L. \& Barton, N. H. Optimality, mutation and the evolution of ageing. Nature 362, 305-311 462 (1993).

463 32. Hughes, K. A. \& Reynolds, R. M. Evolutionary and mechanistic theories of aging. Annu. Rev. 
Entomol. 50, 421-445 (2005).

33. Burnet, M. Intrinsic mutagenesis. (1974) doi:10.1007/978-94-011-6606-5.

466 34. Ellis, P. et al. Reliable detection of somatic mutations in solid tissues by laser-capture

467 microdissection and low-input DNA sequencing. Nat. Protoc. 16, 841-871 (2021).

468 35. Alexandrov, L. B. et al. Signatures of mutational processes in human cancer. Nature 500, 415-421

469 (2013).

470 36. Alexandrov, L. B. et al. The repertoire of mutational signatures in human cancer. Nature 578, 94-101 (2020).

37. Gori, K. \& Baez-Ortega, A. sigfit: flexible Bayesian inference of mutational signatures. bioRxiv 372896 (2020) doi:10.1101/372896.

38. Lindahl, T. \& Nyberg, B. Heat-induced deamination of cytosine residues in deoxyribonucleic acid. Biochemistry 13, 3405-3410 (1974).

39. Zou, X. et al. A systematic CRISPR screen defines mutational mechanisms underpinning signatures caused by replication errors and endogenous DNA damage. Nat Cancer 2, 643-657 (2021).

40. Wilson, M. R. et al. The human gut bacterial genotoxin colibactin alkylates DNA. Science 363, (2019).

41. Pleguezuelos-Manzano, C. et al. Mutational signature in colorectal cancer caused by genotoxic pks+ E. coli. Nature 580, 269-273 (2020).

42. Smati, M. et al. Quantitative analysis of commensal Escherichia coli populations reveals hostspecific enterotypes at the intra-species level. Microbiologyopen 4, 604-615 (2015).

43. Oliero, M. et al. Oligosaccharides increase the genotoxic effect of colibactin produced by pks + Escherichia coli strains. BMC Cancer 21, 172 (2021). 1029-1041.e21 (2017). univariate longevity measures using zoological animal records. Aging Cell 11, 940-948 (2012). 
46. Tidière, M. et al. Comparative analyses of longevity and senescence reveal variable survival benefits of living in zoos across mammals. Sci. Rep. 6, 36361 (2016).

47. Speakman, J. R. Correlations between physiology and lifespan--two widely ignored problems with comparative studies. Aging Cell 4, 167-175 (2005).

48. de Magalhães, J. P., Costa, J. \& Church, G. M. An analysis of the relationship between metabolism, developmental schedules, and longevity using phylogenetic independent contrasts. J. Gerontol. A Biol. Sci. Med. Sci. 62, 149-160 (2007).

49. Risques, R. A. \& Promislow, D. E. L. All's well that ends well: why large species have short telomeres. Philos. Trans. R. Soc. Lond. B Biol. Sci. 373, (2018).

50. Tollis, M., Schneider-Utaka, A. K. \& Maley, C. C. The Evolution of Human Cancer Gene Duplications across Mammals. Mol. Biol. Evol. 37, 2875-2886 (2020).

51. Vazquez, J. M. \& Lynch, V. J. Pervasive duplication of tumor suppressors in Afrotherians during the evolution of large bodies and reduced cancer risk. Elife 10, (2021).

52. Buffenstein, R. Negligible senescence in the longest living rodent, the naked mole-rat: insights from

54. Millar, J. S. \& Zammuto, R. M. Life Histories of Mammals: An Analysis of Life Tables. Ecology 64, $631-635$ (1983).

510 55. Speakman, J. R. Body size, energy metabolism and lifespan. J. Exp. Biol. 208, 1717-1730 (2005).

511 56. Sanders, M. A. et al. Life without mismatch repair. bioRxiv 2021.04.14.437578 (2021) doi:10.1101/2021.04.14.437578.

513 57. Lane, R. K., Hilsabeck, T. \& Rea, S. L. The role of mitochondrial dysfunction in age-related 514 diseases. Biochim. Biophys. Acta 1847, 1387-1400 (2015).

515 58. Kauppila, T. E. S., Kauppila, J. H. K. \& Larsson, N.-G. Mammalian Mitochondria and Aging: An 
$516 \quad$ Update. Cell Metab. 25, 57-71 (2017).

517 59. Ju, Y. S. et al. Origins and functional consequences of somatic mitochondrial DNA mutations in 518 human cancer. Elife 3, (2014).

519 60. Hall, K. Y., Hart, R. W., Benirschke, A. K. \& Walford, R. L. Correlation between ultraviolet-induced

520 DNA repair in primate lymphocytes and fibroblasts and species maximum achievable life span.

$521 \quad$ Mech. Ageing Dev. 24, 163-173 (1984).

522 61. Tian, X. et al. SIRT6 Is Responsible for More Efficient DNA Double-Strand Break Repair in Long$523 \quad$ Lived Species. Cell 177, 622-638.e22 (2019).

524 62. Zhang, L. et al. Maintenance of genome sequence integrity in long- and short-lived rodent species. $525 \quad$ Sci $A d v$ 7, eabj3284 (2021).

526 63. Smith, J. M. Review Lectures on Senescence - I. The causes of ageing. Proceedings of the Royal $527 \quad$ Society of London. Series B. Biological Sciences 157, 115-127 (1962).

528 64. Jaiswal, S. et al. Age-related clonal hematopoiesis associated with adverse outcomes. N. Engl. J. $529 \quad$ Med. 371, 2488-2498 (2014).

530 65. Martincorena, I. et al. Somatic mutant clones colonize the human esophagus with age. Science 362, $5319911-917(2018)$.

532 66. Yokoyama, A. et al. Age-related remodelling of oesophageal epithelia by mutated cancer drivers. $533 \quad$ Nature 565, 312-317 (2019).

534 67. Jaiswal, S. \& Ebert, B. L. Clonal hematopoiesis in human aging and disease. Science 366, (2019).

535 68. Ren, A. A. et al. PIK3CA and CCM mutations fuel cavernomas through a cancer-like mechanism. $536 \quad$ Nature 594, 271-276 (2021).

537 69. Jaiswal, S. \& Libby, P. Clonal haematopoiesis: connecting ageing and inflammation in $538 \quad$ cardiovascular disease. Nat. Rev. Cardiol. 17, 137-144 (2020).

539 70. Ng, S. W. K. et al. Convergent somatic mutations in metabolism genes in chronic liver disease.

$540 \quad$ Nature 598, 473-478 (2021).

541 71. Swovick, K. et al. Interspecies Differences in Proteome Turnover Kinetics Are Correlated With Life 
542 Spans and Energetic Demands. Mol. Cell. Proteomics 20, 100041 (2021).

543 72. Whittemore, K., Vera, E., Martínez-Nevado, E., Sanpera, C. \& Blasco, M. A. Telomere shortening

544 rate predicts species life span. Proc. Natl. Acad. Sci. U. S. A. 116, 15122-15127 (2019).

545 73. Li, H. Aligning sequence reads, clone sequences and assembly contigs with BWA-MEM. arXiv [q546 bio.GN] (2013).

547 74. Jones, D. et al. cgpCaVEManWrapper: Simple Execution of CaVEMan in Order to Detect Somatic

548 Single Nucleotide Variants in NGS Data. Curr. Protoc. Bioinformatics 56, 15.10.1-15.10.18 (2016).

549 75. Raine, K. M. et al. cgpPindel: Identifying Somatically Acquired Insertion and Deletion Events from $550 \quad$ Paired End Sequencing. Curr. Protoc. Bioinformatics 52, 15.7.1-15.7.12 (2015).

551 76. Gerstung, M., Papaemmanuil, E. \& Campbell, P. J. Subclonal variant calling with multiple samples $552 \quad$ and prior knowledge. Bioinformatics 30, 1198-1204 (2014).

553 77. Gerstung, M. et al. Reliable detection of subclonal single-nucleotide variants in tumour cell

554 populations. Nat. Commun. 3, 811 (2012).

555 78. Quinlan, A. R. \& Hall, I. M. BEDTools: a flexible suite of utilities for comparing genomic features.

556 Bioinformatics 26, 841-842 (2010).

557 79. Tacutu, R. et al. Human Ageing Genomic Resources: new and updated databases. Nucleic Acids Res.

558 46, D1083-D1090 (2018).

559 80. Conde, D. A. et al. Data gaps and opportunities for comparative and conservation biology. Proc.

$560 \quad$ Natl. Acad. Sci. U. S. A. 116, 9658-9664 (2019).

561 81. Blokzijl, F., Janssen, R., van Boxtel, R. \& Cuppen, E. MutationalPatterns: comprehensive genome-

562 wide analysis of mutational processes. Genome Med. 10, 33 (2018).

563 82. Kumar, S., Stecher, G., Suleski, M. \& Hedges, S. B. TimeTree: A Resource for Timelines, Timetrees, 564 and Divergence Times. Mol. Biol. Evol. 34, 1812-1819 (2017).

565 83. Snippert, H. J. et al. Intestinal crypt homeostasis results from neutral competition between

$566 \quad$ symmetrically dividing Lgr5 stem cells. Cell 143, 134-144 (2010).

567 84. Rijke, R. P., Plaisier, H. M. \& Langendoen, N. J. Epithelial cell kinetics in the descending colon of 
the rat. Virchows Arch. B Cell Pathol. Incl. Mol. Pathol. 30, 85-94 (1979).

569

570

571

572

573

574

575

576

577

578

579

580

581

582

583

584

585

586

587

588

589

590

591

592

593

594

595

596

597

85. Potten, C. S., Kellett, M., Rew, D. A. \& Roberts, S. A. Proliferation in human gastrointestinal epithelium using bromodeoxyuridine in vivo: data for different sites, proximity to a tumour, and polyposis coli. Gut 33, 524-529 (1992).

86. Bach, S. P., Renehan, A. G. \& Potten, C. S. Stem cells: the intestinal stem cell as a paradigm. Carcinogenesis 21, 469-476(2000).

\section{Acknowledgements}

We are grateful to the animals whose tissues were used in this study. We thank the staff of Wellcome Sanger Institute Sample Logistics, Sequencing and Informatics facilities for their contribution; CASM IT and administrative staff for their help processing the data. This research was made possible by the worldwide information network of zoos and aquariums, which are members of Species360 and is authorized by Species360 research data use and grant agreement \#60633. We thank Dalia Conde and Johanna Staerk for help accessing Species360 data.

\section{Funding}

This research was funded by Wellcome (grant number 206194). For the purpose of Open Access, the author has applied a CC BY public copyright licence to any Author Accepted Manuscript version arising from this submission.

\section{Author Contributions}

A.C., E.P.M., M.R.S. and I.M. conceived the project. I.M., E.P.M., and M.R.S. supervised the project. E.F., S.S., I.J., E.W., N.M., R.D., M.W.P., M.D., R.B., M.D.M., F.G., F.C.-C., L.P., D.B., E.St.J.S., B.N., and E.P.M. performed and facilitated sample collection. A.C. performed the laser capture microdissection. A.C., L.M.R.H., A.R.J.L., Y.H., K.R., E.A., S.L., M.M, P.S., J.F., L.O., and C.L processed the samples. A.C., A.B.-O., F.A., N.B., T.H.H.C., M.A.S., D.J., R.A. and K.R processed the data. J.S. and D.C provided animal longevity records. A.C., A.B.-O. and N.B. led the analysis with help from F.A., T.H.H.C., M.A.S., A.R.J.L., T.M.B., T.D., H.J., E.P.M. and I.M. The manuscript was written by A.C., A.B.-O., N.B. and I.M. with input from all the authors. 
bioRxiv preprint doi: https://doi.org/10.1101/2021.08.19.456982; this version posted December 14,2021 . The copyright holder for this preprint (which was not certified by peer review) is the author/funder, who has granted bioRxiv a license to display the preprint in perpetuity. It is made available under aCC-BY-NC-ND 4.0 International license.

598

599 Competing Interests

600 The authors declare no competing interests.

601 
Figure 1. Somatic mutation burden in mammalian colorectal crypts. a, Histology images from horse, lion, naked mole-rat and rat colon samples, with one colorectal crypt marked in each. b, Burden of somatic single-base substitutions and indels per genome in each colorectal crypt sample (corrected for the size of the analysable genome). Samples are grouped by individual, with samples from the same individual coloured in the same shade. Species, and individuals within each species, are sorted by mean mutation burden $\mathbf{c}$, Linear regression of somatic substitution burden (corrected for analysable genome size) on individual age for dog, human, mouse and naked mole-rat samples.

611

612

613 Samples from the same individual are shown in the same colour. Regression was performed using 614 mean mutation burden per individual. Shaded areas indicate $95 \%$ confidence intervals of the regression line. BW: black-and-white, $\mathrm{H}$ : harbour, N: naked, RT: ring-tailed.

a
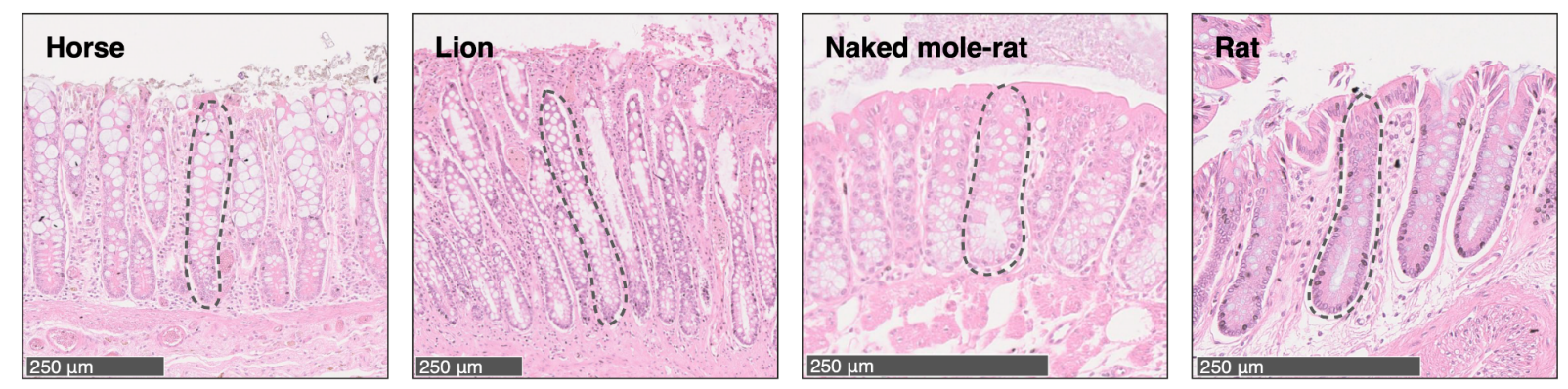

b Substitutions Indels

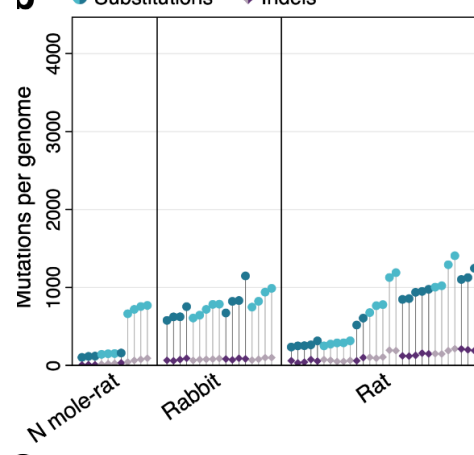

$c$
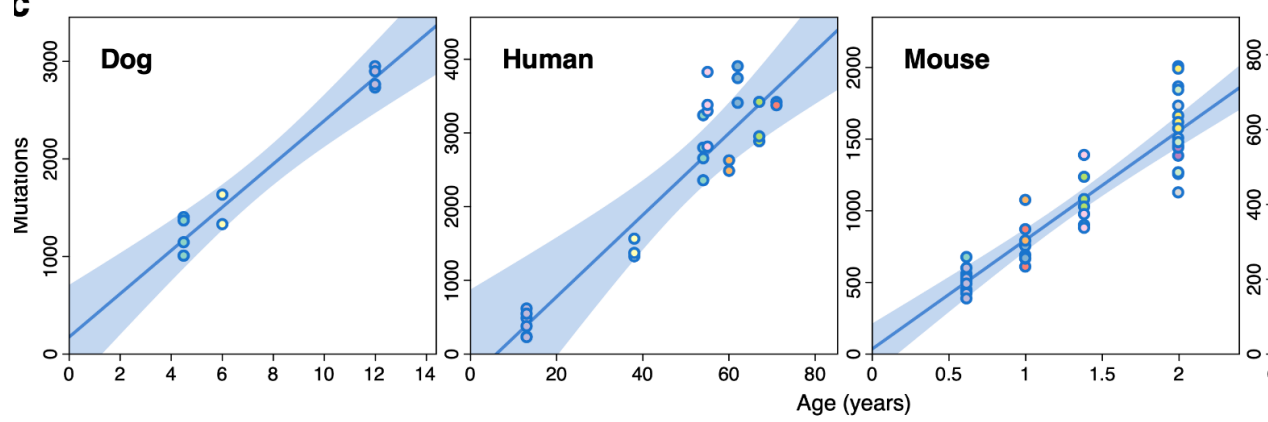
618 Figure 2. Mutational processes in the mammalian colon. a, Mutational spectra of somatic single-base substitutions in each species. The $x$-axis presents 96 mutation types on a trinucleotide context, coloured by base substitution type. b, Mutational signatures inferred from (SBSB, SBSC) or fitted to (SBS1) the species mutational spectra shown in a, and normalised to the human genome trinucleotide frequencies. c, Estimated contribution of each signature to each sample. Samples are arranged horizontally as in Fig. 1b. d, Regression of signature-specific mutation burdens on individual age for human, mouse and naked mole-rat samples. Regression was performed using 626 mean mutation burden per individual. Shaded areas indicate 95\% confidence intervals of the regression line. BW, black-and-white; $\mathrm{H}$, harbour; N, naked; RT, ring-tailed.
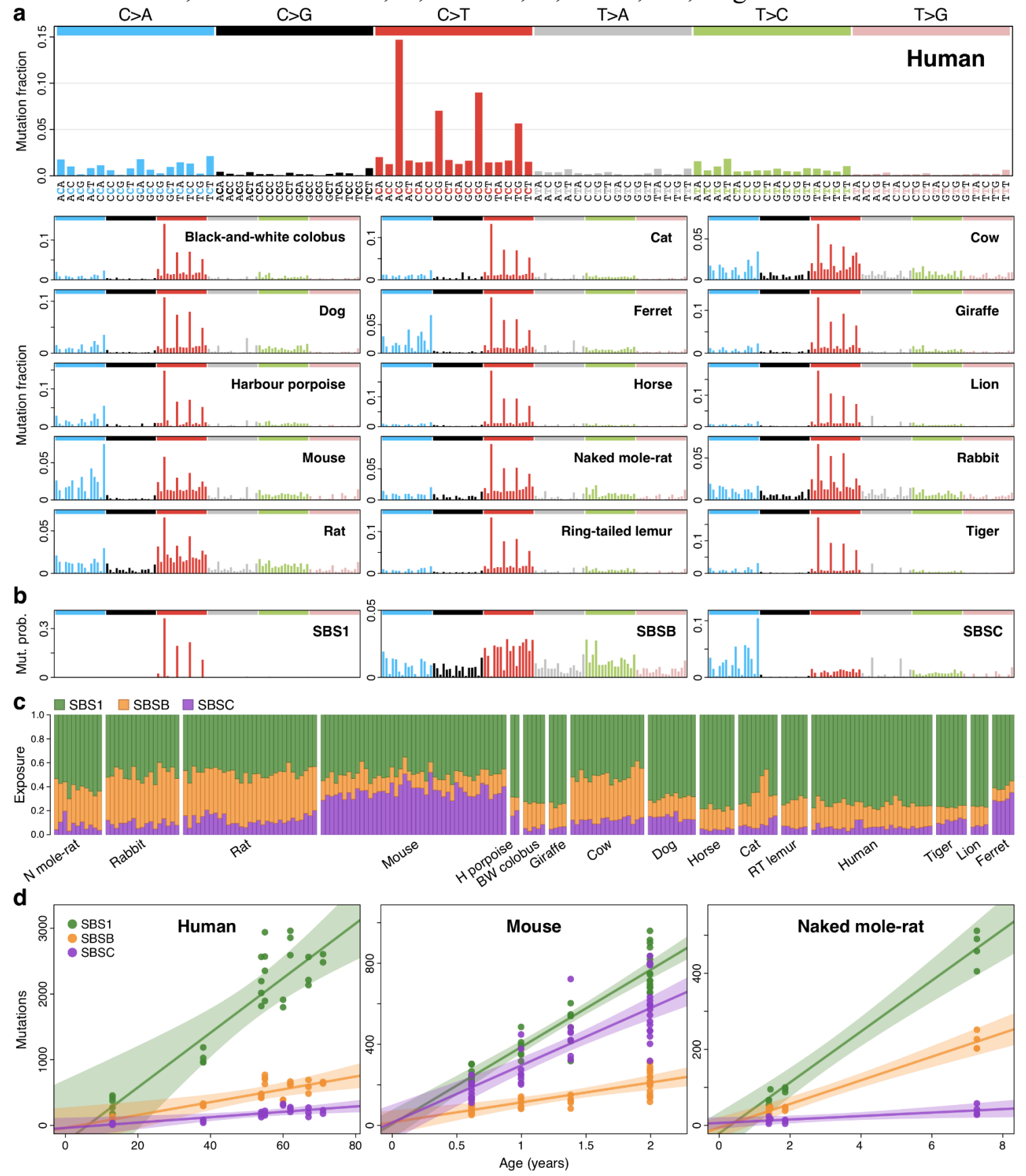

628 Figure 3. Associations between somatic mutation rates and life-history variables. a, Somatic mutation rate per year (top) and expected end-of-lifespan substitution burden in each crypt.

630 Samples are grouped and sorted as in Fig. 1b; harbour porpoise samples were excluded due to the 
631 unknown age of the individual. b, Allometric (log-log) regression of somatic mutation rate on 632 lifespan (left), and regression of body-mass-adjusted residuals for somatic mutation rates and 633 lifespan (partial correlation, Methods). Regressions were performed using a simple linear model 634 on the mean mutation rate per species. Shaded areas represent $95 \%$ confidence intervals $(\mathrm{CI})$ of the 635 regression lines. Fraction of inter-species variance explained by the model (FVE) and $p$-value $(P)$ 636 are indicated (note that for simple linear regression $\mathrm{FVE}=R^{2}$ ). Dashed line denotes a reference 637 slope of -1 . c, Zero-intercept linear mixed-effects (LME) regression of somatic mutation rate on 638 inverse lifespan (1/lifespan), presented on the scale of untransformed lifespan ( $x$-axis). For 639 simplicity, the $y$-axis represents mean mutation rate per species, although mutation rates per crypt 640 were used in the regression. Darker shaded area indicates 95\% CI of the regression line; lighter 641 shaded area marks a two-fold deviation from the regression line. The point estimate and $95 \% \mathrm{CI}$ 642 of the slope $(k)$, the FVE, and the range of end-of-lifespan burden (ELB) are shown. d, Allometric 643 regression and linear regression of lifespan-adjusted residuals for somatic mutation rate and body 644 mass. Elements are as described in b. e, Free-intercept LME regression of somatic mutation rate 645 on log-transformed adult mass. The $y$-axis represents mean mutation rate per species, although 646 mutation rates per crypt were used in the regression. Shaded area indicates the 95\% bootstrap 647 interval of the regression line. Point estimates of the regression intercept and slope coefficients, 648 and model FVE, are indicated. f, Comparison of FVE values for free-intercept LME models using 649 1/lifespan and other life-history variables (alone or in combination with 1/lifespan) as explanatory 650 variables. Error bars indicate $95 \%$ bootstrap intervals. BMR, basal metabolic rate; BW, black-and651 white; N, naked; RT, ring-tailed. 
bioRxiv preprint doi: https://doi.org/10.1101/2021.08.19.456982; this version posted December 14, 2021. The copyright holder for this preprint (which was not certified by peer review) is the author/funder, who has granted bioRxiv a license to display the preprint in perpetuity. It is made available under aCC-BY-NC-ND 4.0 International license.
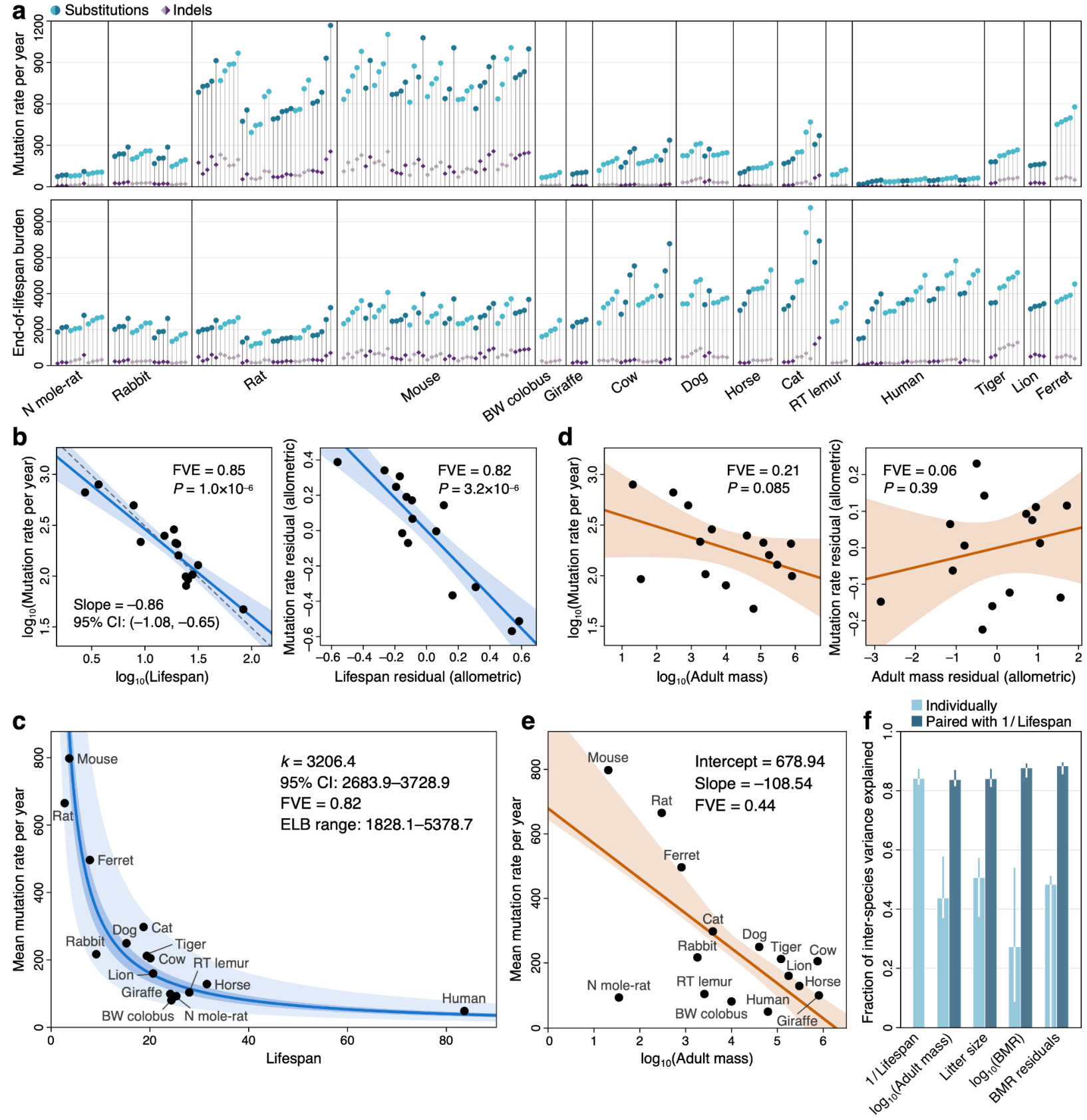
Figure 4. Association between mutation rate subtypes and species lifespan. Zerointercept linear mixed-effects regression of somatic rates of signature-specific substitutions, indels and mtDNA mutations (top to bottom) on inverse lifespan (1/lifespan), presented on the scale of untransformed lifespan ( $x$-axis). For simplicity, $y$-axes represent mean mutation rate per species, although mutation rates per crypt were used in the regressions. Darker shaded areas indicate $95 \%$ confidence intervals (CI) of the regression lines; lighter shaded areas mark a two-fold deviation from the regression lines. Point estimates of the regression slope coefficient $(k)$, fraction of inter-species variance explained by each model (FVE), and ranges of end-of-lifespan burden (ELB) are shown.
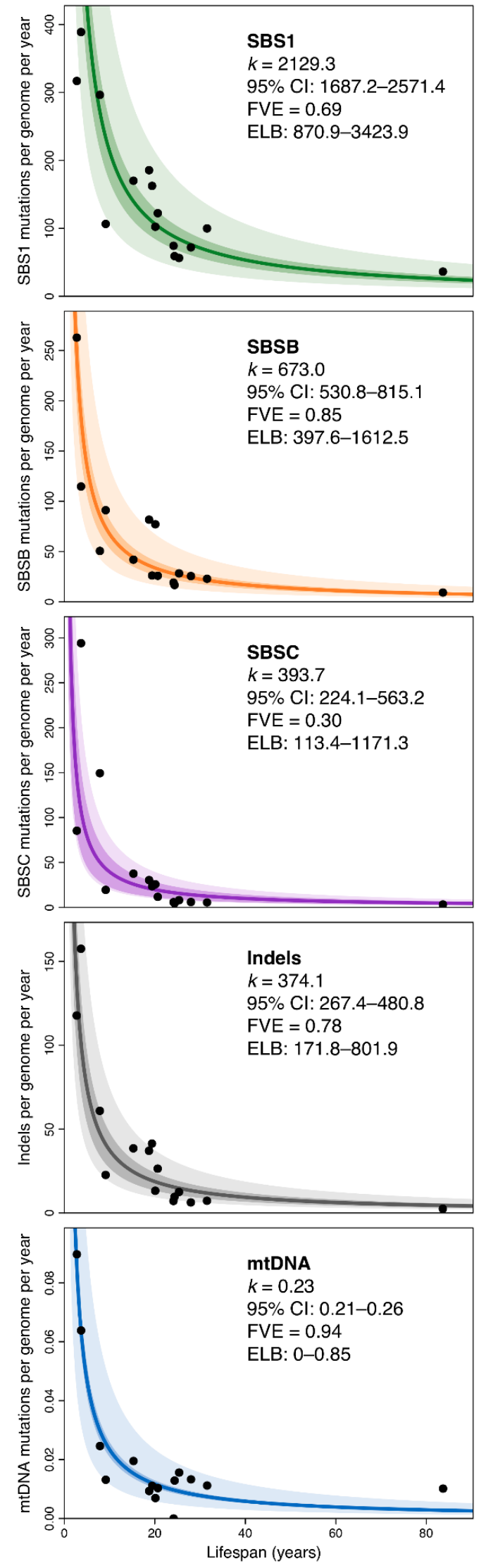


\section{Tables}

Table 1. Variation in adult mass, lifespan, mutation rate and end-of-lifespan burden across the 16 mammalian species surveyed. Species-level estimates are provided in Supplementary Tables 3 and 6 .

\begin{tabular}{|l|l|l|l|}
\hline Variable & Minimum & Maximum & Fold variation \\
\hline Adult mass (g) & 20.50 & $800,000.00$ & $39,024.39$ \\
\hline Lifespan (yr) & 2.75 & 83.67 & 30.44 \\
\hline $\begin{array}{l}\text { Mutation rate per year } \\
\text { (SBS/genome) }\end{array}$ & 47.12 & 796.42 & 16.90 \\
\hline $\begin{array}{l}\text { End-of-lifespan burden } \\
\text { (SBS/genome) }\end{array}$ & 1828.08 & 5378.73 & 2.94 \\
\hline
\end{tabular}


METHODS

\section{1. Sample collection}

4 We obtained colorectal epithelium and skin samples from a range of sources (Supplementary

5 Table 1). For comparability across species a $\sim 1 \mathrm{~cm}$ biopsy of the colorectal epithelium was taken

6 from the terminal colon during necropsy. All necropsies occurred as soon as possible post-

7 mortem to minimise tissue and DNA degradation. Tissue samples taken later than 24 hours post-

8 mortem typically showed extensive degradation of the colorectal epithelium, making

9 identification of colorectal crypts challenging. These samples were also associated with poor

10 DNA yields and so were not included in his study. Sampled tissue was fixed in PAXgene FIX

11 (PreAnalytiX, Hombrechtikon, Switzerland), a commercially available fixative, during the

12 necropsy. After 24 hours in the fixative at room temperature samples were transferred into the

13 PAXgene STABILIZER and stored at $-20^{\circ} \mathrm{C}$ until further processing.

\section{2 . Sample processing}

16 Samples were processed using a workflow designed for detection of somatic mutations in solid

17 tissues by laser-capture microdissection (LCM) using low-input DNA sequencing. For a more

18 detailed description see the paraffin workflow described in Ellis et al ${ }^{34}$. Briefly, PAXgene-fixed

19 tissue samples of the colorectal epithelium were paraffin-embedded using a Sakura Tissue-Tek

20 VIP tissue processor. Sections of $16 \mu \mathrm{m}$ were cut using a microtome, mounted on PEN-

21 membrane slides, and stained with Gill's haematoxylin and eosin by sequential immersion in the

22 following: xylene (two minutes, twice), ethanol (100\%, 1 minute, twice), deionised water (1

23 minute, once), Gill's haematoxylin (10 seconds, once), tap water (20 seconds, twice), eosin (5

24 seconds, once), tap water (20 seconds, once), ethanol (70\%, 20 seconds, twice) and xylene or

25 Neo-Clear, a xylene substitute (20 seconds, twice).

27 High-resolution scans were obtained from representative sections of each species. Example

28 images are shown in Fig. 1a and Extended Data Figure 2. Individual colorectal crypts were

29 isolated from sections on polyethylene naphthalate (PEN) membrane slides by laser-capture

30 microdissection with a Leica LMD7 microscope. Haematoxylin and eosin histology images were

31 reviewed by a veterinary pathologist. For some samples we also cut a section of muscle tissue

32 from below the colorectal epithelium of the section to use as a germline control for variant calling 
33 (Supplementary Table 2). Pre- and post-microdissection images of the tissue were recorded for

34 each crypt and muscle sample taken. Each microdissection was collected in a separate well of a

35 96-well plate.

37 Crypts were lysed using the Arcturus PicoPure Kit (Applied Biosystems) as previously

38 described ${ }^{8,34}$. Each crypt then underwent DNA library preparation, without a quantification step

39 to avoid loss of DNA, following the protocol described in Ellis et al. ${ }^{34}$. For some animals a

40 PAXgene fixed bulk skin biopsy was used as the germline control. For these skin samples, DNA

41 was extracted using the DNeasy Blood \& Tissue Kit (Qiagen).

\section{Library preparation and sequencing}

44 Libraries from microdissected samples were prepared using enzymatic fragmentation, adapter

45 ligation and whole-genome sequencing following the method described in Ellis et al. ${ }^{34}$. Libraries

46 from skin samples were prepared using standard Illumina whole-genome library preparation.

47 Samples were multiplexed and sequenced using Illumina XTEN and Novaseq 6000 machines to

48 generate 150 base pair (bp) paired-end reads. Samples were sequenced to $\sim 30 \times$ depth

49 (Supplementary Table 2).

\section{Sequence read alignment}

52 For each species sequences were aligned to a reference assembly (Supplementary Table 2)

53 using the BWA-MEM algorithm ${ }^{73}$ as implemented in BWA version 0.7.17-r1188, with options "-

54 T $30-Y-p-t$ 8'. The aligned reads were sorted using the bamsort tool from the biobambam2

55 package, version 2.0.86 (gitlab.com/german.tischler/biobambam2), with options 'fixmates=1

56 level $=1$ calmdnm $=1$ calmdnmrecompindetonly $=1$ calmdnmreference $=<$ reference_fasta $>$

57 outputthreads $=7$ sortthreads $=7$ '. Duplicate reads were marked using the bammarkduplicates 2 tool

58 from biobambam2, with option 'level=0'.

\section{Variant calling}

61 Identification of somatic single-base substitutions (SBS) and short insertions and deletions

62 (indels) was divided into two steps: variant calling, and variant filtering to remove spurious calls

63 (see 'Variant filtering' below). For human colorectal crypts, we obtained previously sequenced

64 and mapped reads from a study where colorectal crypts were isolated by laser-capture 
65 microdissection $^{8}$, and processed them using the sample variant calling and filtering process that

66 was applied to the non-human samples.

68 Substitutions were identified using the Cancer Variants through Expectation Maximization

69 (CaVEMan) algorithm ${ }^{74}$, version 1.13.15. CaVEMan uses a naive Bayesian classifier to perform

70 a comparative analysis of the sequence data from a target and control sample from the same

71 individual to derive a probabilistic estimate for putative somatic substitutions at each site. The

72 copy number options were set to 'major copy number $=5$ ' and 'minor copy number $=2$ ', as in

73 our experience this maximises the sensitivity to detect substitutions in normal tissues. CaVEMan

74 identifies and excludes germline variants shared in the target (colorectal crypt) and matched

75 normal (skin or muscle tissue) samples, and produces a list of putative somatic mutations present

76 only in the target sample. CaVEMan was run separately for each colorectal crypt, using either

77 bulk skin or muscle microdissected from the sample colorectal biopsy as the matched normal

78 control (Supplementary Table 2). For two human donors where an alternative tissue was not

79 available, a colonic crypt not included as a target sample was used as the matched normal control.

81 Indels were identified using the Pindel algorithm ${ }^{75}$, version 3.3.0, using a second sample from the

82 same individual as a matched control. The indel calls produced by Pindel were subsequently re-

83 genotyped using the vafCorrect tool (github.com/cancerit/vafCorrect), which performs a local

84 sequence assembly to address alignment errors for indels located at the end of sequence reads,

85 and produces corrected counts of sequence reads supporting the indel and corrected estimates of

86 variant allele fraction (VAF).

\section{Variant filtering}

89 A number of post-processing filters were applied to the variant calls to remove false positives

90 (Extended Data Figure 17a,b).

91 Quality flag filter. CaVEMan and Pindel annotate variant calls using a series of quality flags,

92 with the 'PASS' flag denoting no quality issues affecting the call ${ }^{74,75}$. Variant calls presenting

93 any flag other than 'PASS' were discarded.

94 Alignment quality filter. Variants were excluded if more than half of the supporting reads were

95 clipped. The library preparation methods create short insert size libraries that can result in reads

96 overlapping. To avoid the risk of double-counting mutant reads we used fragment-based 
statistics. Variants without at least four high-quality fragments (alignment score $\geq 40$ and base

Phred quality score $\geq 30$ ) were excluded. Variants were excluded if reads supporting the variant had a secondary alignment score that was greater than the primary alignment score. This filter was not applied to indel calls.

101 Hairpin filter. To remove variants introduced by erroneous processing of cruciform DNA during

102 the enzymatic digestion we applied a custom filter to remove variants in inverted repeats ${ }^{34}$. This

103 filter was not applied to indel calls.

104 Chromosome and contig filter. For species with chromosome-level assemblies, we discarded

105 variants located in non-chromosomal contigs, including the mitochondrial genome (calling of

106 mitochondrial variants is described in the section 'Mitochondrial variant calling and filtering').

107 For males, variants on the Y chromosome were excluded for species where the Y chromosome

108 was annotated in the assembly.

109 N-tract and contig-end filter. To reduce artefactual calls due to read misalignment, we

110 discarded variants located within 1 kilobase $(\mathrm{kb})$ of a tract of 50 or more consecutive $\mathrm{N}$ bases in

111 the reference assembly, as well as variants within $1 \mathrm{~kb}$ of the start or end of a contig (this implies

112 discarding all variants in contigs shorter than $2 \mathrm{~kb}$ ).

113 Sequencing coverage filter. A sample-specific read depth filter was designed to exclude sites

114 with coverage above the 99th coverage percentile in the sample or its matched normal control, as

115 well as sites with coverage $<10 \times$ in the sample or its matched normal control.

116 Allelic strand bias filter. We discarded variants without any supporting reads on either the

117 forward or reverse strand.

118 Indel proximity filter. We discarded variants for which the total number of reads supporting the 119 presence of an indel within $10 \mathrm{bp}$ of the variant was more than 3 times larger than the number of 120 reads supporting the presence of the variant. This filter was not applied to indel calls.

121 Spatial clustering filter. Visual assessment of variant calls and mutational spectra showed

122 spatially clustered variants to be highly enriched for artefacts. Therefore, we discarded groups of

123 two or more variants located within $1 \mathrm{~kb}$ of each other.

124 Beta-binomial filter. For each crypt, an artefact filter based on the beta-binomial distribution

125 was applied, which exploits read count information in other crypts from the same individual.

126 More specifically, for each sample, we fitted a beta-binomial distribution to the variant allele

127 counts and sequencing depths of somatic variants across samples from the same individual. The

128 beta-binomial distribution was used to determine whether read support for a mutation varies 
across samples from an individual, as expected for genuine somatic mutations but not for

130 artefacts. Artefacts tend to be randomly distributed across samples and can be modelled as drawn

131 from a binomial or a lowly overdispersed beta-binomial distribution. True somatic variants will

132 be present at a high VAF in some samples, but absent in others, and are hence best captured by a

133 highly overdispersed beta-binomial. For each variant site, the maximum likelihood estimate of

134 the overdispersion factor $(\rho)$ was calculated using a grid-based method, with values ranging

135 between $10^{-6}$ and $10^{-0.05}$. Variants with $\rho>0.3$ were considered to be artefactual and discarded.

136 The code for this filter is based on the Shearwater variant caller ${ }^{76}$. We found this to be one of the

137 most effective filters against spurious calls (Extended Data Figure 17b).

138 Minimum VAF filter. For each sample, we discarded variants whose variant allele fraction

139 (VAF) was less than half the median VAF of variants passing the beta-binomial filter (see above)

140 in that sample.

141 Maximum indel VAF filter. For each sample, we discarded indels presenting VAF $>0.9$, as

142 such indels were found to be highly enriched in spurious calls in some species. This filter was not

143 applied to SBS calls.

145 To validate our variant calling strategy, we used LCM to microdissect two sections from the 146 same mouse colorectal crypt. We expected to detect a high fraction of shared somatic variants in 147 these two sections, since their cells should be derived from the same ancestral epithelial stem cell. 148 Both sections were submitted for independent library preparation, genome sequencing, variant 149 calling and filtering using our pipeline. The majority of SBS variant calls (2742 of 2933, 93.5\%)

150 were shared between both sections (Extended Data Figure 17c). In contrast, when comparing 151 five separate crypts from a mouse, a maximum of two variants were shared between two crypts, 152 and no variants were shared by three or more crypts (Extended Data Figure 17d).

\section{Sample filtering}

155 Our method for estimation of mutation rates assumes monoclonality of colorectal crypt samples.

156 This assumption can be violated due to several causes, including contamination from other

157 colorectal crypts during microdissection or library preparation, contamination with non-epithelial

158 cells located in or near the crypt, insufficient time for a stem cell to drift to clonality within the

159 crypt, or the possibility that in some species, unlike in humans ${ }^{8}$, polyclonal crypts are the norm.

160 Therefore, a truncated binomial mixture model was applied in order to remove crypts that showed 
161 evidence of polyclonality, or for which the possibility of polyclonality could not be excluded. An

162 expectation-maximization (EM) algorithm was employed to determine the optimal number of

163 variant allele fraction (VAF) clusters within each crypt sample, as well as each cluster's location

164 and relative contribution to the overall VAF distribution. The algorithm considered a range of

165 numbers of clusters (1-5), with the optimal number being that which minimised the Bayesian

166 Information Criterion (BIC). As the minimum number of supporting reads to call a variant was 4,

167 the binomial probability distribution was truncated to incorporate this minimum requirement for

168 the number of successes, and subsequently re-normalised. The EM algorithm returned the

169 inferred optimal number of clusters, the mean VAF (location) and mixing proportion

170 (contribution) of each clone, and an assignment of each input variant to the most likely cluster.

171 After applying this model to the somatic substitutions identified in each sample, sample filtering

172 was performed on the basis of the following three criteria.

173 Low mutation burden. We discarded samples presenting fewer than 50 somatic variants, which

174 was indicative of low DNA quality or sequencing issues.

175 High mutation burden. We discarded samples with a number of somatic variants greater than

176 three times the median burden of samples from the same individual (excluding samples with less

177 than 50 variants). This served to exclude a small minority of samples presenting evident

178 sequencing quality problems (such as low sequencing coverage), but which did not fulfill the

179 low-VAF criterion for exclusion (see below).

180 Low VAF. We discarded samples in which less than $70 \%$ of the somatic variants were assigned

181 to clusters with VAF $\geq 0.3$. However, this rule was not applied to those cases in which all the

182 samples from the same individual had primary clusters with mean $\mathrm{VAF}<0.3$; this was done to

183 prevent the removal of samples from individuals presenting high fractions of non-epithelial cells,

184 but whose crypts were nonetheless dominated by a single clone.

185 These criteria led to the exclusion of 41 out of 249 samples. On the basis of visual assessment of

186 sequencing coverage and VAF distributions, we decided to preserve three samples

187 (ND0003c_lo0004, ND0003c_lo0011, TIGRD0001b_lo0010) which we considered to be clonal,

188 but which would have been discarded based on the criteria above.

190 8. Mitochondrial variant calling and filtering

191 For six species whose reference genome assemblies did not include the mitochondrial sequence, 192 mitochondrial reference sequences were obtained from the GenBank database (Supplementary 
193 Table 5). For each species, alignment to the reference genome was performed using BWA

194 version 0.7.17-r1188, as described above (see 'Sequence read alignment'). Pileup files were

195 generated for mtDNA genomes using the 'bam2R' function in the deepSNV (v1.32.0) R

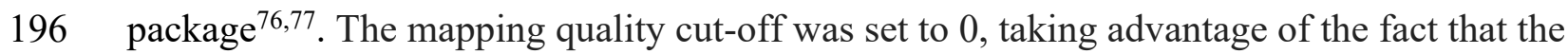

197 mitochondrial genome coverage for most samples was $>100$-fold higher than the nuclear genome

198 coverage, and hence most reads with poor mapping scores should be of mitochondrial origin.

199 Mitochondrial variants were called using the Shearwater algorithm ${ }^{76}$ (deepSNV package

200 v1.32.0). Multiple rounds of filtering were applied to identify and remove false positives. The

201 first set of filters removed germline polymorphisms, applied a maximum false discovery rate

202 (FDR) threshold of $q>0.01$, required that mismatches should be supported by at least one read

203 on both the forward and reverse strands, and merged consecutive indel calls. Further filtering

204 steps were as follows.

205 Minimum VAF filter. Only variants with VAF $>0.01$ were considered for analysis, based on the 206 quality of the mutational spectra.

207 Sequencing coverage filter. Due to species-specific mtDNA regions of poor mappability, we

208 discarded sites with read coverage $<500 \times$.

209 D-loop filter. Analysis of the distribution of mutations along the mitochondrial genome revealed

210 clusters of mutations within the hypervariable region of mtDNA known as the D-loop. To obtain

211 estimates of the mutation burden in mtDNA unaffected by hypermutation of the D-loop,

212 mutations in the D-loop region (coordinates MT:1-576 and MT:16,024-16,569 in human) were

213 excluded from this analysis

214 High mutation burden. We discarded samples having a number of somatic mtDNA variants

215 greater than four times the mean mtDNA burden across all samples. This served to exclude a

216 small minority of samples that were suspected of enrichment in false positive calls. Visual

217 inspection of these samples in a genome browser confirmed the presence of high numbers of

218 variants found on sequence reads with identical start positions and/or multiple base mismatches,

219 suggestive of library preparation or sequencing artefacts.

221 We examined the mutational spectra of somatic mtDNA substitutions on a trinucleotide sequence

222 context (Extended Data Figure 16). The specificity of the filtered variant calls was supported by

223 the observation that the mutational spectra across species were broadly consistent with those 
224 previously observed in studies of human tissues ${ }^{59}$, with a dominance of $\mathrm{C}>\mathrm{T}$ and $\mathrm{T}>\mathrm{C}$

225 transversions and a strong replication strand bias.

\section{Mitochondrial copy number analysis}

228 Sequence reads from each sample were separately mapped to the species-specific mtDNA reference sequence in order to estimate average mtDNA sequencing coverage. Excluding nuclear reference sequences from the alignment enabled obtaining even coverage across the

231 mitochondrial genome by preventing mismapping of sequence reads to inherited nuclear

232 insertions of mitochondrial DNA (known as NuMTs). Next, coverage information from

233 individual mtDNA and whole-genome alignment (BAM) files was obtained using the genomecov 234 tool in the bedtools suite (v2.17.0) ${ }^{78}$. Mitochondrial copy number was calculated according to the 235 formula

$$
\text { depth }_{m t D N A} \times \text { ploidy / depth }{ }_{g D N A},
$$

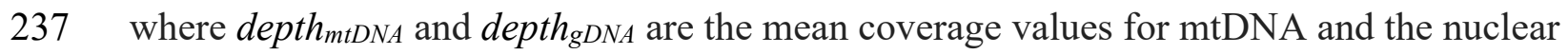
238 genome, respectively, and ploidy $=2$ (assuming normal somatic cells to be diploid). For

239 simplicity, the sex chromosomes were excluded from the calculation of the mean nuclear genome 240 coverage.

\section{Analysable genome size calculation}

242 To estimate the somatic mutation rate, it was first necessary to establish the size of the analysable 243 nuclear genome (i.e. the portion of the genome where variant calling could be performed reliably)

244 for each sample (Supplementary Table 4). For both single-base substitutions and indels, the 245 analysable genome of a sample was defined as the complement of the union of the following 246 genomic regions: regions reported as 'not analysed' by the CaVEMan variant caller; regions 247 failing the 'chromosome and contig' filter; regions failing the 'N-tract and contig-end' filter; and 248 regions failing the 'sequencing coverage' filter (see 'Variant filtering'). For the analysis of 249 mitochondrial variants, the analysable genome of a sample was defined as the portion of mtDNA 250 satisfying the 'sequencing coverage' filter (see 'Mitochondrial variant calling and filtering'), after 251 subtracting the hypervariable region (D-loop).

\section{Life history data}


254 Obtaining accurate lifespan estimates is challenging; while point estimates of maximum lifespan

255 are available for many species, their veracity is often difficult to assess and estimates can vary

256 widely for the same species (Supplementary Table 6). There can be many causes for this

257 variation, including errors in recording and real variation in longevity between populations (i.e.

258 captive versus wild). As we were interested in whether the somatic mutation burden has an

259 association with lifespan in the absence of extrinsic mortality, we sought to obtain estimates of

260 longevity from individuals under human care, to minimise the impact of external factors such as

261 predation or infection.

263 Mortality records for 14 species were obtained from the Species360 database, authorized by

264 Species360 research data use agreement \#60633 [Species360 Zoological Information

265 Management System (ZIMS) (2020), zims.Species360.org]. This database contains lifespan data

266 of zoo animals from international zoo records. Using records from 1980 to the present, we

267 excluded animals for which the date of birth or death was unknown or uncertain. To avoid infant

268 mortality influencing the longevity estimates for each species, we removed animals that died

269 before the age of female sexual maturity, as defined by the AnAge database ${ }^{79}$. This resulted in a

270 mean of 2,681 animal lifespan records per species for the species in the study (minimum 309,

271 maximum 8403; Supplementary Table 6). For the domestic dog, we combined records for

272 domestic dogs (Canis lupus familiaris) and wolves (Canis lupus) because of the paucity of

273 records for domestic dogs in Species360. Although the data are curated, they are still vulnerable

274 to the presence of inaccurate records, which can bias the lifespan estimates. To reduce the impact

275 of these outliers, for each species lifespan was estimated as the age at which $80 \%$ of the adults

276 from that species had died (Supplementary Table 6) ${ }^{46}$.

278 Human longevity estimates were obtained using census birth and death record data from

279 Denmark, (1900-2020), Finland (1900-2019) and France (1900-2018), retrieved from the

280 Human Mortality Database [University of California, Berkeley (USA), and Max Planck Institute

281 for Demographic Research (Germany); www.mortality.org, www.humanmortality.de]. We

282 selected these countries because they had census records going back at least 100 years. To

283 remove the impact of infant mortality, we excluded individuals who died before the age of 13.

284 For each country, we selected the cohort born in 1900 and calculated the age at which $80 \%$ of the

285 individuals had died (Denmark, 87 years; Finland, 83 years; France, 81 years). We then used the 
mean of the three countries as our estimate of the human $80 \%$ lifespan (83.7 years)

(Supplementary Table 6).

To test the impact of different estimates of lifespan on our results, we also obtained maximum longevity estimates for each species from a range of databases ${ }^{80}$ and a survey of the literature

(Supplementary Table 6). Other life-history metrics were obtained from the AnAge database ${ }^{79}$

\section{(Supplementary Table 6).}

293

\section{Mutational signature analysis}

295 Mutational signatures of single-base substitutions on a trinucleotide sequence context were inferred from sets of somatic mutation counts using the sigfit (v2.0) R package ${ }^{37}$. Initially, signature extraction was performed de novo for a range of numbers of signatures $(N=2, \ldots, 10)$, using counts of mutations grouped per sample, per individual and per species. To account for differences in sequence composition across samples, and especially across species, mutational opportunities per sample, per individual and per species were calculated from the reference trinucleotide frequencies across the analysable genome of each sample (see 'Analysable genome size calculation'), and supplied to the 'extract_signatures' function in sigfit. The 'convert_signatures' function in sigfit was subsequently used to transform the extracted signatures to a human-relative representation (Fig. 2 b), by scaling the mutation probability values using the corresponding human genome trinucleotide frequencies. The best-supported number of signatures, on the basis of overall goodness-of-fit ${ }^{37}$ and consistency with known COSMIC signatures (cancer.sanger.ac.uk/signatures), was found to be $N=3$. The cleanest deconvolution of the three signatures was achieved when using the mutation counts grouped by species, rather than by sample or individual. The three extracted signatures (labelled SBSA, SBSB, SBSC) were

310 found to be highly similar to COSMIC signatures SBS1 (cosine similarity 0.96), SBS5 (0.89),

311 and SBS18 (0.91), respectively. These signatures were independently validated using the

312 MutationalPatterns (v1.12.0) R package ${ }^{81}$, which produced comparable signatures (respective

313 cosine similarities $0.999,0.98$ and 0.89$)$.

315 This de novo signature extraction approach, however, failed to deconvolute signatures SBSA and

316 SBSB entirely from each other, resulting in a general overestimation of the exposure to SBSA

317 (Extended Data Figure 18). To obtain more accurate estimates of signature exposure, the 
318 deconvolution was repeated using an alternative approach that combines signature fitting and

319 extraction in a single inference process ${ }^{37}$. More specifically, the 'fit_extract_signatures' function

320 in sigfit was used to fit COSMIC signature SBS1 (retrieved from the COSMIC v3.0 signature

321 catalogue; cancer.sanger.ac.uk/signatures) to the mutation counts grouped by species (with

322 species-specific mutational opportunities), while simultaneously extracting two additional

323 signatures de novo (SBSB and SBSC). Before this operation, COSMIC SBS1 was transformed

324 from its human-relative representation to a genome-independent representation using the

325 'convert_signatures' function in sigfit. By completely deconvoluting SBS1 and SBSB, this

326 approach yielded a version of SBSB that was more similar to COSMIC SBS5 (cosine similarity

327 0.93); the similarity of SBSC to COSMIC SBS18 was the same under both approaches (0.91).

329 Finally, the inferred signatures were re-fitted to the mutational spectra of mutations in each

330 sample (using the 'fit_signatures' function in sigfit with sample-specific mutational

331 opportunities) to estimate the exposure of each sample to each signature. The fitting of the three

332 signatures yielded spectrum reconstruction similarity values (measured as the cosine similarity

333 between the observed mutational spectrum and a spectrum reconstructed from the inferred

334 signatures and exposures) with median 0.98 and interquartile range 0.96-0.99. Although the

335 purely de novo extraction approach and the 'fitting and extraction' approach yielded comparable

336 versions of signatures SBSB and SBSC, the fixing of COSMIC SBS1 in the latter approach

337 resulted in lower SBS1 exposures and higher SBSB exposures in the majority of samples, due to

338 the cleaner deconvolution of these two signatures (Fig. 2, Extended Data Figure 18).

340 To examine potential variation in the spectrum of signature SBS5 across species, the following

341 procedure was conducted for each species: individual-specific mutation counts and mutational

342 opportunities were calculated for each individual in the species, and the 'fit_extract_signatures'

343 function was used to fit COSMIC signatures SBS1, SBS18 and SBS34 (transformed to a

344 genome-independent representation using the 'convert_signatures' function) to the mutational

345 spectra of each individual, while simultaneously inferring one additional signature

346 (corresponding to signature SBS5 as manifested in that species; Extended Data Figure 6).

348 To assess the presence in non-human colorectal crypts of mutational signatures caused by

349 APOBEC or colibactin, which have been previously observed in human crypts $^{8}$, we used an 
350 expectation-maximisation algorithm for signature fitting, in combination with likelihood ratio

351 tests (LRTs). More specifically, for each non-human sample, we tested for exposure to colibactin

352 (signature SBS88, COSMIC v3.2) by comparing the log-likelihoods of $(i)$ a model fitting

353 COSMIC signatures SBS1, SBS5, SBS18, SBS34 and SBS88, and (ii) a reduced model fitting

354 only the first four signatures. Benjamini-Hochberg multiple-testing correction was applied to the

$355 p$-values resulting from the LRTs, and colibactin exposure was considered significant in a sample

356 if the corresponding corrected $q$-value was less than 0.05 . We followed the same approach to

357 assess exposure to APOBEC (SBS2 and SBS13), using two separate sets of LRTs for models

358 including either SBS2 or SBS13, in addition to SBS1, SBS5, SBS18 and SBS34. APOBEC

359 exposure was considered significant in a sample if its $q$-values for the models including SBS2

360 and SBS13 were both less than 0.05 . This analysis identified 1/180 crypts with significant

361 exposure to each of colibactin and APOBEC (although the evidence for the presence of the

362 relevant signatures in these two crypts was not conclusive). To test for depletion of colibactin or

363 APOBEC exposure in non-human crypts relative to human crypts, we first applied the LRT-

364 based method described above to a published set of 445 human colorectal crypts ${ }^{8}$, identifying 92

365 colibactin-positive and 9 APOBEC-positive crypts. We then compared the numbers of colibactin-

366 and APOBEC-positive crypts in the human and non-human sets using two separate Fisher's exact

367 tests ('fisher.test' function in R). This revealed the difference in colibactin exposure to be highly

368 significant $\left(P=7 \times 10^{-14}\right)$, unlike the difference in APOBEC exposure $(P=0.30)$.

370 Mutational spectra of somatic indels identified in each species were generated using the

371 'indel.spectrum' function in the Indelwald tool for R (24/09/2021 version;

372 github.com/MaximilianStammnitz/Indelwald).

\section{13. Selection analysis}

375 Evidence of selection was assessed using the ratio of nonsynonymous to synonymous substitution

376 rates $(\mathrm{dN} / \mathrm{dS})$ in the somatic mutations called in each species. The $\mathrm{dNdScv}(\mathrm{v} 0.0 .1 .0) \mathrm{R}_{\text {package }}{ }^{44}$

377 was used to estimate $\mathrm{dN} / \mathrm{dS}$ ratios for missense and truncating substitutions in each species

378 separately. Reference CDS databases for the dNdScv package were built for those species with

379 available genome annotation in Ensembl (www.ensembl.org; Supplementary Table 2), using

380 the 'buildref' function in dNdScv. For each species, the 'dndscv' function was applied to the list

381 of somatic substitutions called in samples of that species, after de-duplicating any substitutions 
382 that were shared between samples from the same individual in order to avoid counting shared

383 somatic mutations multiple times. In addition, the analysis was restricted to genes that were fully

384 contained in the analysable genomes of all samples from the species (a condition satisfied by the

385 vast majority of protein-coding genes). Genome-wide and gene-specific $\mathrm{dN} / \mathrm{dS}$ ratios were

386 obtained for missense and truncating substitutions in each species; no genes with statistically

387 significant $\mathrm{dN} / \mathrm{dS} \neq 1$ were observed.

\section{14. Copy number analysis}

390 For species with chromosome-level assemblies (cat, cow, dog, horse, human, mouse, rabbit, rat), 391 total and allele-specific copy number $(\mathrm{CN})$ were assessed in each sample adapting a likelihood

392 model previously applied to the detection of subclonal CN changes in normal human skin ${ }^{13}$. This 393 method exploits two sources of evidence: relative sequencing coverage and B-allele fraction

394 (BAF; the fraction of reads covering a heterozygous SNP that support one of the alleles). Human 395 samples PD36813x15 and PD36813x16 were excluded from this analysis due to the poor quality 396 of their SNP data.

398 For each sample, sequencing coverage was measured in non-overlapping 100-kilobase (kb) bins

399 along the species' reference genome, using the coverageBed tool in the bedtools suite (v2.17.0 $)^{78}$.

400 For each bin, the coverage per base pair was calculated by dividing the number of reads mapping 401 to the bin by the bin length, and multiplying the result by the read length (150 bp). A normalised

402 sample-normal coverage ratio was then calculated for each bin by dividing the bin coverage in

403 the sample by the corresponding coverage in its matched normal control (see 'Sample

404 processing'). Heterozygous SNPs were isolated for each sample by selecting germline SNPs with

405 a BAF between 0.4 and 0.6 in the matched normal sample, and a coverage of at least 15 reads in

406 both the target sample and its matched normal sample. After assigning each SNP to its

407 corresponding 100-kb genome bin, the bins in each sample were divided into two sets: $(i)$ bins

408 with coverage $\geq 10$ in both the target sample and its matched normal, and at least one

409 heterozygous SNP; and (ii) bins with coverage $\geq 10$ in both the target sample and its matched

410 normal, and no heterozygous SNPs. For the first set, estimates of total and allele-specific CN

411 were inferred by maximising the joint likelihood of a beta-binomial model for BAF and a

412 negative binomial model for relative coverage, as previously described ${ }^{13}$. The most likely

413 combination of allele $\mathrm{CN}$ values was obtained for each bin by conducting an exhaustive search of 
$414 \mathrm{CN}$ values between 0 and 4, and selecting the combination maximising the joint likelihood

415 (calculated on the basis of expected BAF and relative coverage values). A penalty matrix was

416 used to penalise more complex solutions over simpler ones, as previously described ${ }^{13}$. For the

417 second set of bins (bins without SNPs), only estimates of total CN were inferred, by maximising

418 the likelihood of a negative binomial model for relative coverage. The most substantial

419 differences between these methods and the one previously published are: $(i)$ SNPs were obtained

420 from the variant calling output, instead of from a public database; ( $i$ i) relative coverage was

421 calculated per 100-kb bin, rather than per SNP; (iii) SNPs were not phased within each gene, but

422 within each bin; (iv) no reference bias was assumed (i.e. the underlying BAF of heterozygous

423 SNPs was assumed to be 0.5$)$; (v) the minimum sample purity was raised to 0.85 ; (vi) putative

$424 \mathrm{CN}$ changes were not subjected to significance testing, but selected according to their likelihood,

425 and subsequently filtered by means of a segmentation algorithm (see below).

427 Estimates of total and allele-specific CN per bin were merged into CN segments, which were

428 defined as contiguous segments composed of five or more bins with identical CN states.

429 Segmentation was performed separately for total and allele-specific CN estimates in each sample.

430 After this process, any pair of adjacent segments with the same CN assignment, and separated by

431 a distance shorter than 5 bins, was merged into a single segment. Finally, within each species,

432 segments presenting $\mathrm{CN}$ values other than 2 (or $1 / 1$ for allele-specific $\mathrm{CN}$ ), and being either

433 shorter than 10 bins (1 megabase), or shared among two or more samples, were discarded,

434 resulting in the removal of nearly all spurious $\mathrm{CN}$ changes.

\section{Mutation rate estimation}

437 For each sample, the somatic mutation density (mutations per bp) was calculated by dividing the

438 somatic mutation burden (total number of mutations called) by the analysable genome size for the

439 sample (see 'Analysable genome size calculation'). The adjusted somatic mutation burden

440 (number of mutations per whole genome) was then calculated by multiplying the mutation

441 density by the total genome size of the species (see below). The somatic mutation rate per year

442 (mutations per genome per year) was obtained by dividing this adjusted mutation burden by the

443 age of the individual, expressed in years (Supplementary Table 2). The expected end-of-

444 lifespan burden (ELB) for each sample was calculated by multiplying the somatic mutation rate

445 by the estimated lifespan of the species (see 'Life history data'). 
447 The total genome size of a species was estimated as the total size of its reference genome

448 assembly. Across species, the mean genome size was 2.67 gigabases (Gb), ranging between 2.41

$449 \mathrm{~Gb}$ and $3.15 \mathrm{~Gb}$ and with a standard deviation of 221 megabases (Supplementary Table 4). This

450 suggests that inter-species variation in genome size should not have a substantial influence on the

451 somatic mutation rate estimates. For an assessment of alternative measures of mutation rate, see

452 'Association of mutation rate and end-of-lifespan burden with lifespan'.

453

\section{16. Association of mutation rate with life-history traits}

455 The association of the somatic mutation rate with different life-history traits was assessed using

456 linear mixed-effects (LME) models. In particular, associations with the following traits were

457 examined: lifespan (in years), adult mass (or adult weight, in grams), basal metabolic rate (BMR,

458 in watts), and litter size (see 'Life history data'). Associations for lifespan, adult mass and BMR

459 were assessed using the following transformed variables: 1/Lifespan, $\log _{10}$ (Adult mass), and

$460 \log _{10}(\mathrm{BMR})$. To account for the potentially confounding effect of the correlation between

461 metabolic rate and body mass, the residuals of a fitted allometric regression model of BMR on

462 adult mass (equivalent to a simple linear regression of $\log _{10}(\mathrm{BMR})$ on $\log _{10}$ (Adult mass)) were

463 employed as a mass-adjusted measure of metabolic rate, referred to as 'BMR residuals'.

465 For each variable, an LME model was implemented for the regression of somatic mutation rates

466 per sample on the variable of interest, using the 'lme' function in the nlme R package (v3.1-137;

467 cran.r-project.org/package=nlme). To account for non-independence of the samples, both at the

468 individual level and at the species level, the model included fixed effects (intercept and slope

469 parameters) for the variable of interest, and random effects (slope parameters) at the individual

470 and species levels. In addition, to account for the heteroscedasticity of mutation rate estimates

471 across species, the usual assumption of constant response variance was replaced with explicit

472 species-specific variances, to be estimated within the model.

474 To determine the fraction of inter-species variance in mutation rate explained by each life-history

475 variable individually, the LME model described above was used to produce predictions of the

476 mean mutation rate per species; only fixed effects were employed when obtaining these

477 predictions, random effects being ignored. The variance of these predictions was then compared 
to the variance in observed mean mutation rates; the latter were calculated for each species as the mean of the observed mean rates per individual, to avoid individuals with larger numbers of samples exerting a stronger influence on the species mean. The fraction of inter-species variance explained by the model was calculated using the standard formula for the coefficient of determination,

$$
R^{2}=\mathrm{ESS} /(\mathrm{ESS}+\mathrm{RSS})
$$

where ESS is the explained sum of squares, and RSS is the residual sum of squares:

$$
\mathrm{ESS}=\sum_{i}\left(\hat{y}_{i}-\bar{y}\right)^{2}, \quad \mathrm{RSS}=\sum_{i}\left(y_{i}-\hat{y}_{i}\right)^{2} .
$$

In this formulation, $y_{i}$ and $\hat{y}_{i}$ denote the observed and predicted mutation rates for species $i$, respectively, and $\bar{y}$ is the overall mean rate. This definition of $R^{2}$ coincides with the fraction of variance explained (FVE), defined as 1 minus the fraction of variance unexplained (FVU):

$$
\mathrm{FVE}=1-\mathrm{FVU}=1-[\mathrm{RSS} /(\mathrm{ESS}+\mathrm{RSS})]=\mathrm{ESS} /(\mathrm{ESS}+\mathrm{RSS})=R^{2} .
$$

As the predicted and observed values correspond to mean mutation rates per species, rather than

mutation rates per sample, FVE provides a measure of the fraction of inter-species variance explained by the fixed effects of the LME model. Among the variables considered, 1/Lifespan was found to have the greatest explanatory power $(\mathrm{FVE}=0.84$, using a free-intercept model).

To compare the explanatory power of variables other than 1/Lifespan when considered either individually or in combination with 1/Lifespan, the method described above was also applied to two-variable combinations of 1/Lifespan and each of the remaining variables, using an LME model with fixed effects for both variables and random effects for 1/Lifespan only. The $R^{2}$ formula above was used to measure the fraction of inter-species variance explained by each model. In addition, to test whether the inclusion of a second explanatory variable was justified by the increase in model fit, likelihood ratio tests between each two-variable LME model and a reduced LME model including only 1/Lifespan were performed using the 'anova' function in the

505 To further assess the potential effects of body mass and lifespan on each other's association with the somatic mutation rate, allometric regression models (equivalent to simple linear models under

507 logarithmic transformation of both variables) were fitted to the mean somatic mutation rate per

508 species, using either adult mass or lifespan as the explanatory variable. In addition, the 
510 allometric regressions among these three variables) were used to examine the associations

511 between somatic mutation rate and either body mass or lifespan, after accounting for the effect of

512 the third variable (partial correlation analysis). For instance, to account for the potential influence

513 of body mass on the relationship between somatic mutation rate and lifespan, the residuals of an

514 allometric regression between mutation rate and adult mass, and the residuals of an allometric

515 regression between lifespan and adult mass, were analysed using simple linear regression. This

516 analysis supported a strong association between somatic mutation rate and lifespan

517 (independently of the effect of mass; FVE=0.82, $P=3.2 \times 10^{-6}$; Fig. 3c), and a non-significant

518 association between somatic mutation rate and body mass (independently of the effect of

519 lifespan). Therefore, the relationship between somatic mutation rate and lifespan does not appear

520 to be mediated by the effect of body mass on both variables. Importantly, this result remains after

521 excluding naked mole-rat: after removing this species, partial correlation analysis still reveals a

522 strong association between somatic mutation rate and lifespan $\left(\mathrm{FVE}=0.77, P=4.1 \times 10^{-5}\right)$, and a

523 non-significant association between somatic mutation rate and body mass $(P=0.84)$. This

524 demonstrates that the observed relationships are not dependent on the presence of naked mole-rat

525 in the study.

527 To assess the robustness of the LME regression analyses described above, we performed

528 bootstrap analysis on each LME model, at the level of both individuals and species. More

529 specifically, for each level we used each of the LME models to perform regression on 10,000

530 bootstrap replicates, produced by resampling either species or individuals with replacement. We

531 then assessed the distributions of FVE across bootstrap replicates (Extended Data Figure 14c).

532 Additionally, we performed a similar bootstrap analysis using a collection of maximum longevity

533 estimates obtained from the literature (see section 'Life history data'). We applied the zero-

534 intercept LME model described above (for regressing mutation rate on inverse lifespan) on a set

535 of 5,000 bootstrap replicates, each of which used a set of species lifespan estimates randomly

536 sampled from the collection of literature-derived estimates (Extended Data Figure 13).

538 The results obtained with the LME models were additionally validated using an independent

539 hierarchical Bayesian model, in which the mean somatic mutation burden of each individual was

540 modelled as following a normal distribution with mean defined as a linear predictor containing a

541 species-specific slope parameter and a multiplicative offset (corresponding to the individual's 
542 age; inclusion of this offset minimises the heteroscedasticity of the mutation rate across species,

543 which results from dividing mutation burdens by age). Species-specific slope parameters were in

544 turn modelled as normally distributed around a global slope parameter, equivalent to the fixed-

545 effect slope estimated by the LME model. This hierarchical model produced very similar results

546 to those of the LME model for all life-history variables (Extended Data Figure 14a).

548 We note that samples CATD0002b_lo0003 and MD6267ab_lo0003 were excluded from all

549 regression analyses, due to the fact that each shared the majority of its somatic variants with

550 another sample from the same individual (indicating, in each case, that both samples were closely

551 related), hence violating the assumption of independence among samples. The inclusion of these

552 two samples, however, had no effect on the outcome of the analyses.

\section{Association of mutation rate and end-of-lifespan burden with lifespan}

555 The relationship between somatic mutation rate and species lifespan was further explored by

556 adapting the LME model described in the previous section to perform constrained (zero-

557 intercept) regression of the adjusted mutation rate per year on the inverse of lifespan, 1/Lifespan

558 (see 'Life history data', 'Mutation rate estimation' and 'Association of mutation rate with life-

559 history traits'). The use of zero-intercept regression was motivated by the prediction that, if

560 somatic mutation is a determinant of maximum lifespan, then it would be expected for all species

561 to end their lifespans with a similar somatic mutation burden. Indeed, this was confirmed via

562 simple linear regression of the species mean end-of-lifespan mutation burden against species

563 lifespan (slope $P=0.39$ ). Thus, if $m$ is the mutation rate per year, and $L$ is the species' lifespan, the

564 expected relationship is of the form

$$
m L \approx k,
$$

566 where $k$ is a constant representing the typical end-of-lifespan mutation burden across species.

567 According to this relationship, the mutation rate per year is linearly related to the inverse of

568 lifespan,

$$
m \approx k(1 / L) .
$$

570 Therefore, the cross-species average end-of-lifespan burden $(k)$, can be estimated as the slope

571 parameter of a zero-intercept linear regression model with the mutation rate per year $(m)$ as the

572 dependent variable, and the inverse of lifespan $(1 / L)$ as the explanatory variable. To this purpose,

573 the LME model described in the previous section was altered by removing the fixed-effect 
574 intercept parameter, thus considering only fixed- and random-effect slope parameters for

$5751 /$ Lifespan.

577 The zero-intercept LME model estimated a value of $k=3210.52$ (95\% confidence interval

578 2686.89-3734.15). The fraction of inter-species variance explained by the zero-intercept model

579 (FVE) was 0.82, while the LME model described in the previous section (which estimated $k=$

5802869.98 , and an intercept of 14.76) achieved FVE $=0.84$ (see 'Association of mutation rate with

581 life-history traits'). To test whether the increase in model fit justifies the inclusion of an intercept,

582 both models were compared using a likelihood ratio test (as implemented by the 'anova' function

583 in the nlme R package [v3.1-137]). This yielded $P=0.23$, indicating that the free-intercept model

584 does not achieve a significantly better fit than the zero-intercept model. Similarly, the zero-

585 intercept model yielded lower values for both the Bayesian information criterion (BIC) and the

586 Akaike information criterion (AIC). Notably, equivalent analyses using somatic mutation rates

587 per megabase and per protein-coding exome (instead of per whole genome) yielded comparable

588 results (Extended Data Figure 12).

590 To investigate the possibility of phylogenetic relationships between the species sampled

591 confounding the analysis, a phylogenetic generalised linear model was used to regress the mean

592 mutation rate of each species on the inverse of its lifespan $(1 / L)$, while accounting for the

593 phylogenetic relationships among species. A phylogenetic tree of the 15 species examined was

594 obtained from the TimeTree resource ${ }^{82}$, and the phylogenetic linear model was fitted using the

595 'pgls' function in the caper R package (v1.0.1; cran.r-project.org/package=caper). The estimates

596 produced by zero-intercept regression of mean mutation rates per species on 1/Lifespan were

597 compared between this phylogenetic generalised linear model and a simple linear model (' $1 \mathrm{~m}$ '

598 function in R). The use of this simple model, as well as the use of mean mutation rates per

599 species (rather than mutation rates per sample), was necessary due to the impossibility of

600 replicating the heteroscedastic mixed-effects structure of the LME model employed for the main

601 association analyses (see 'Association of mutation rate with life-history traits') within the

602 phylogenetic linear model. Both the phylogenetic linear model and the simple linear model

603 produced similar estimates (Extended Data Figure 14b), suggesting that phylogenetic non-

604 independence of the samples does not have a substantial effect on the association analyses. 


\section{Cell division analysis}

607 To investigate the extent to which differences in cell division rate could explain differences in

608 mutation rate and burden across species, we obtained estimates of intestinal crypt cell division 609 rates from mouse ${ }^{83}$, rat $^{84}$ and human ${ }^{85,86}$ (Supplementary Table 7). Using these cell division

610 rates, our lifespan estimates and the observed SBS mutation rates, we calculated the number of

611 cell divisions at the end of lifespan and the corresponding number of mutations per cell division

612 expected under a simple model assuming that all mutations occur during cell division

613 (Supplementary Table 7).

615 We investigated whether differences in the number of cell divisions among species could explain

616 the observed differences in mutation burden. Although colorectal cell division rate estimates are

617 lacking for most species, existing estimates from mouse, rat and human indicate that the total

618 number of stem cell divisions per crypt in a lifetime varies greatly across species, for example

619 with $\sim 6-31$ fold more divisions per intestinal stem cell in a human than a rat over their respective

620 lifetimes, depending on the cell division rate estimates used (Supplementary Table 7). Mouse

621 intestinal stem cells are estimated to divide once every 24 hours ${ }^{83}$, while estimates of the human

622 intestinal stem cell division rate vary from once every 48 hours $^{85}$ to once every 264 hours ${ }^{86}$. Thus

623 mouse intestinal stem cells divide 2-11 times faster than human intestinal stem cells. By the end

624 of lifespan, an intestinal stem cell is predicted to have divided $\sim 1351$ times in a mouse, $\sim 486$

625 times in a rat and 2774-15,257 times in a human (depending on the cell division rate estimate

626 used). Applying our somatic mutation burden and lifespan data, this implies that the somatic

627 mutation rate per cell division in a mouse is $\sim 1.5-8.4$ fold higher than in a human. However, the

628 observed fold difference in somatic mutation rate between these two species is 16.9 (Table 1).

629 Therefore, differences in cell division rate appear unable to fully account for the observed

630 differences in mutation rate across species. Nevertheless, we note that accurate cell division rate

631 estimates for basal intestinal stem cells are lacking for the majority of species.

19. Data reporting

635 No statistical methods were used to predetermine sample size. The experiments were not

636 randomised and the investigators were not blinded to allocation during experiments and outcome 637 assessment. 


\section{20. Ethics statement}

640 All animal samples were obtained with the approval of the local ethical review committee

641 (AWERB) at the Wellcome Sanger Institute and those at the holding institutions.

\section{21. Data and code availability}

644 DNA sequence data have been deposited in the European Genome-Phenome Archive (ega-

645 archive.org) under overarching accession EGAD00001008032. The computer code and data files

646 used in the analyses are available on Zenodo (doi.org/10.5281/zenodo.5554801;

647 doi.org/10.5281/zenodo.5554777) and GitHub (github.com/baezortega/CrossSpecies2021). 
bioRxiv preprint doi: https://doi.org/10.1101/2021.08.19.456982; this version posted December 14,2021 . The copyright holder for this preprint (which was not certified by peer review) is the author/funder, who has granted bioRxiv a license to display the preprint in perpetuity. It is made available under aCC-BY-NC-ND 4.0 International license.

\section{Extended Data Figures}

\section{Extended Data Figure 1. Somatic mutational spectra of the human colon and small}

659 intestine. Trinucleotide-context mutational spectra of somatic single-base substitutions from

660 human adult stem cells in colon (top) and small intestine, using mutation calls obtained from

661 Blokzijl et al. ${ }^{7}$.

662
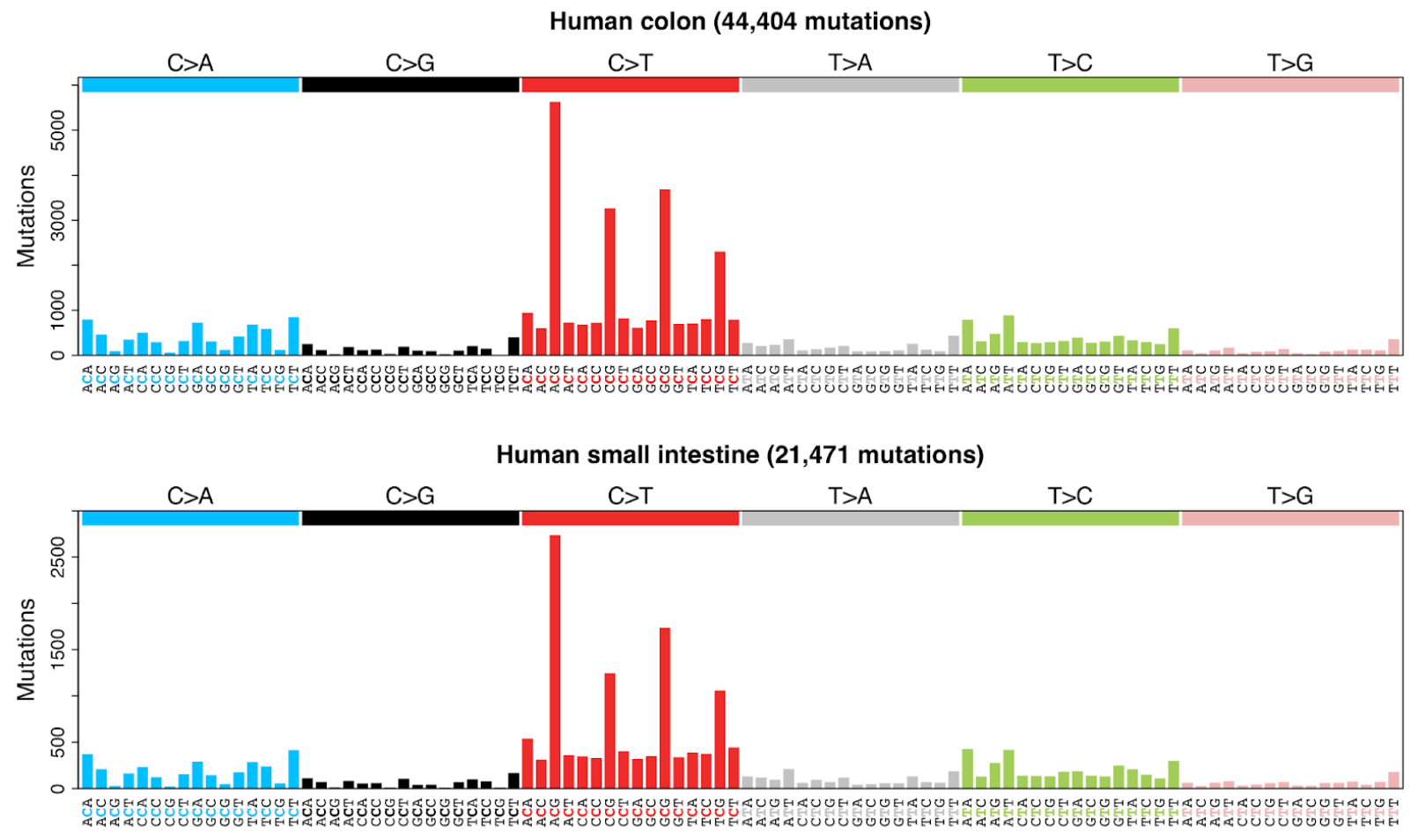
bioRxiv preprint doi: https://doi.org/10.1101/2021.08.19.456982. this version posted December 14.2021. The copyright holder for this preprint (which was not certified by peer review) is the author/funder, who has granted bioRxiv a license to display the preprint in perpetuity. It is made available under aCC-BY-NC-ND 4.0 International license.

666 Extended Data Figure 2. Histology images of intestinal crypts across species. Histological

667 images of the colorectal or intestinal (ferret) epithelium for each non-human species. Scale bars

668 are provided at the bottom of each image.

669
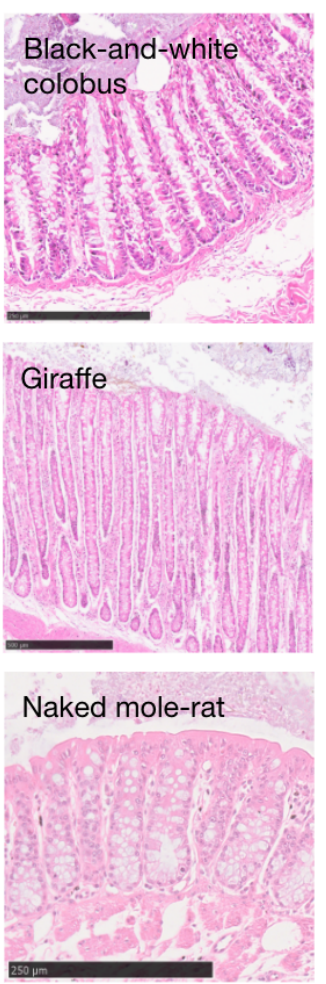
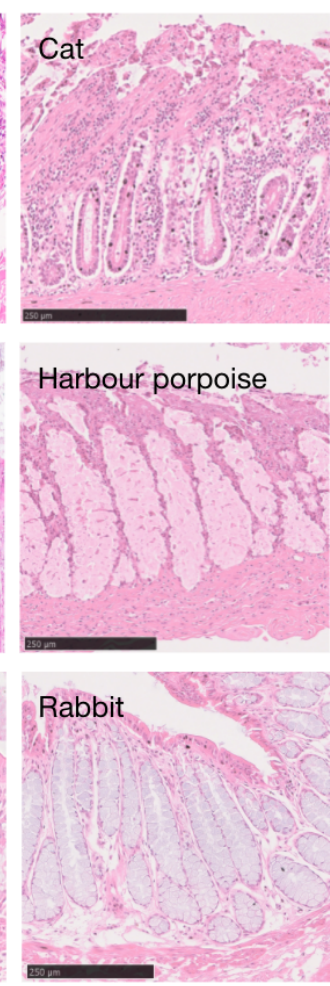
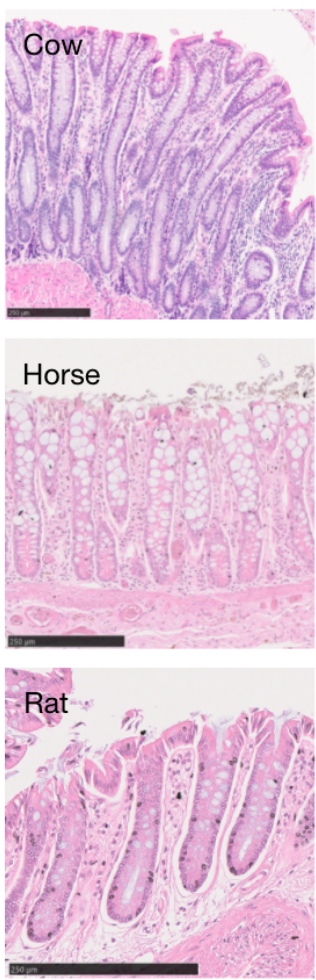
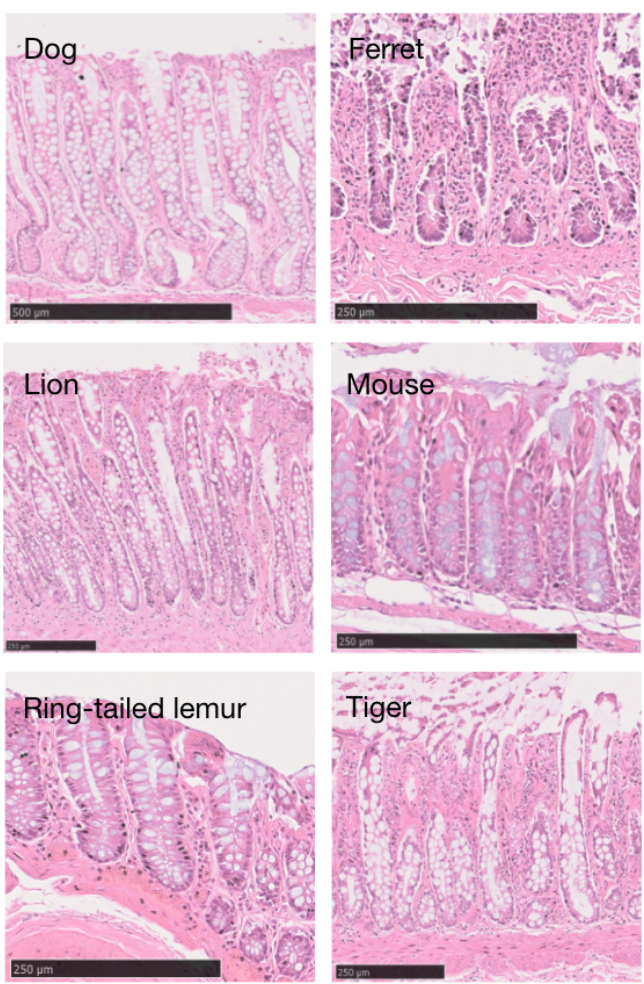

671

672 
674 Extended Data Figure 3. Somatic VAF distributions per species. Distributions of variant

675 allele fraction (VAF) for somatic substitutions in each crypt for each species. Each distribution

676 refers to the variants in a single sequenced crypt.

677
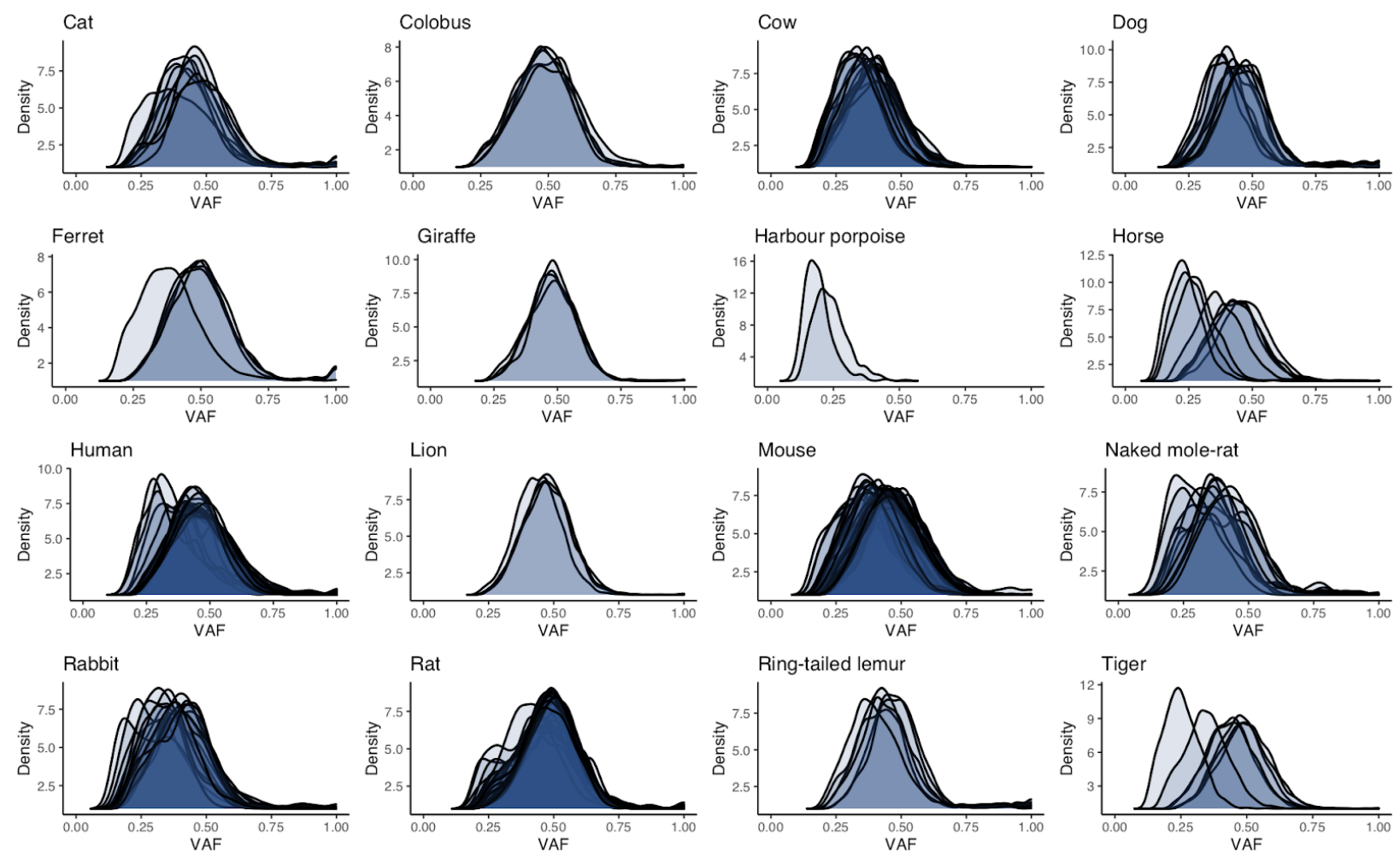
680 Extended Data Figure 4. Somatic mutation accumulation across species. Each panel presents

681 somatic substitution burdens per genome for a given species. Each dot represents a crypt sample,

682 with samples from the same individual sharing the same colour. For species with two or more

683 individuals, the estimated regression line from a simple linear regression model on individual

684 mean burdens is shown. For species with three or more individuals, blue shaded regions indicate

685 the $95 \%$ confidence intervals of the regression line. Harbour porpoise samples were excluded

686 because the individual was of unknown age.

687
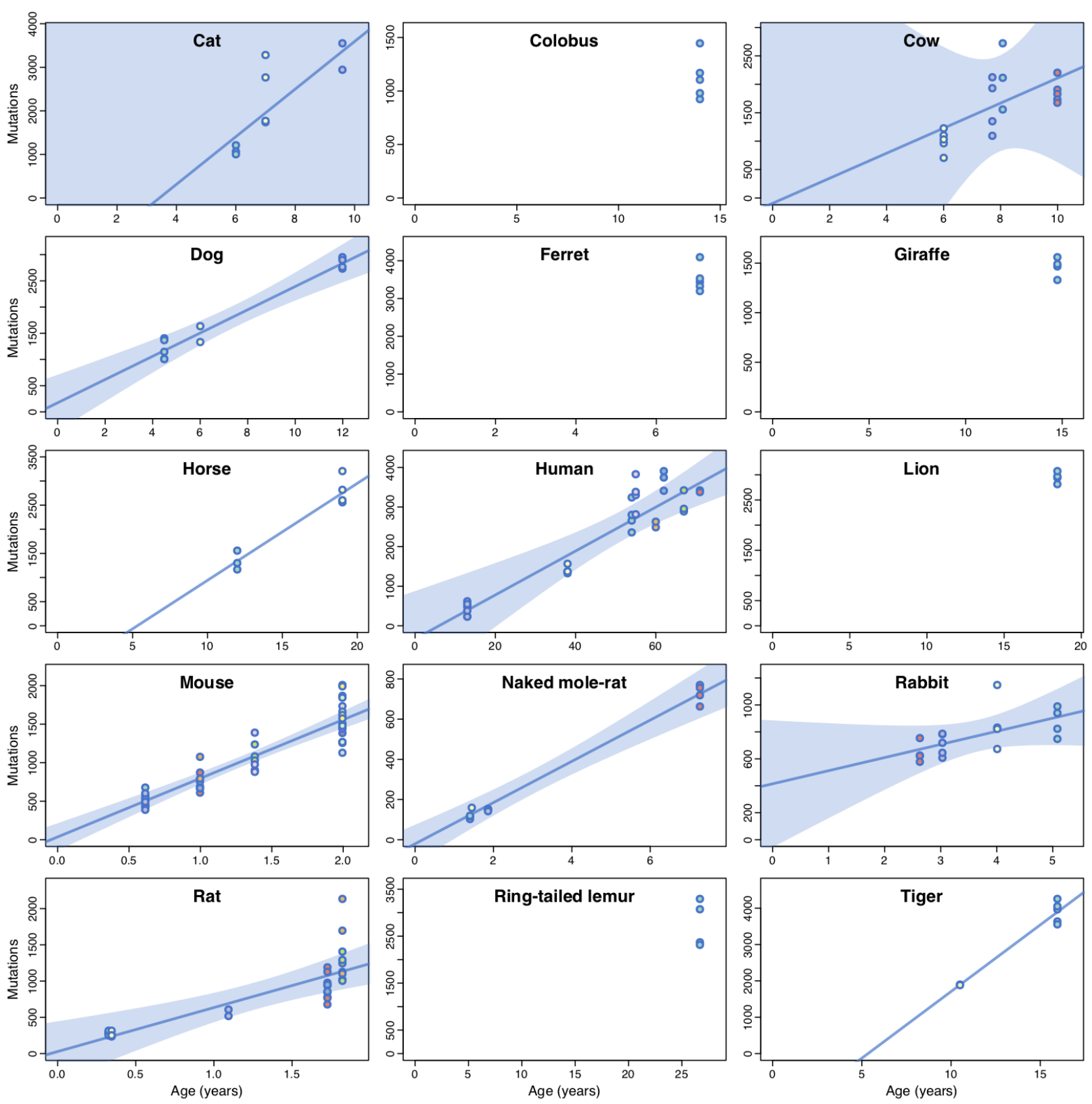
Extended Data Figure 5. Signature-specific mutation accumulation across species. Each

690 panel presents somatic substitution burdens per genome for mutational signatures SBS1 (green),

691 SBSB (yellow) and SBSC (purple) in a given species. For species with two or more individuals,

692 the estimated regression lines from a simple linear regression model on individual mean burdens

693 per signature are shown. For species with three or more individuals, shaded regions indicate the

$69495 \%$ confidence intervals of the regression lines. Harbour porpoise samples were excluded

695 because the individual was of unknown age.

696
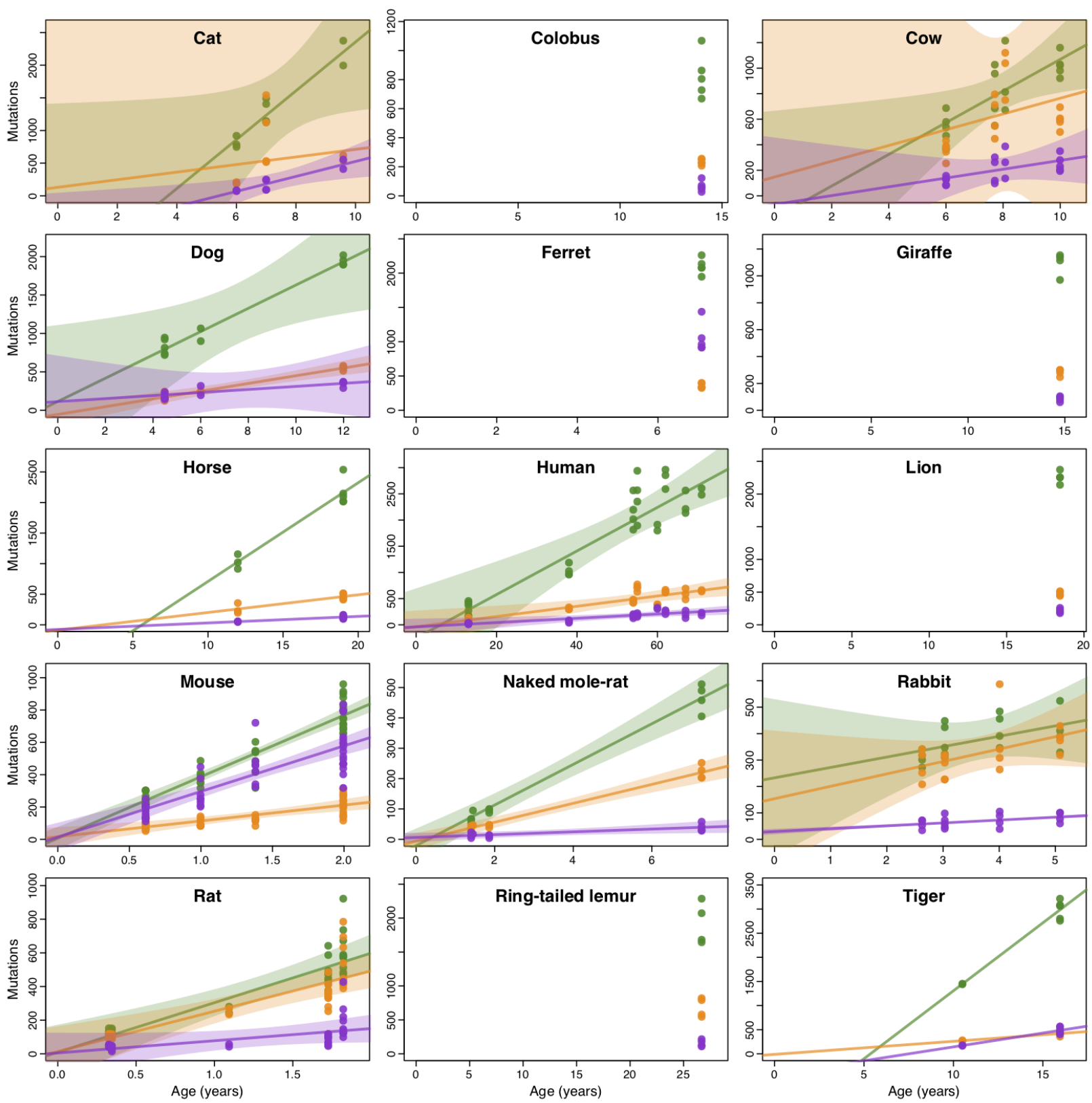
bioRxiv preprint doi: https://doi.org/10.1101/2021.08.19.456982; this version posted December 14,2021 . The copyright holder for this preprint (which was not certified by peer review) is the author/funder, who has granted bioRxiv a license to display the preprint in perpetuity. It is made available under aCC-BY-NC-ND 4.0 International license.

698 Extended Data Figure 6. Profiles of signature SBSB as inferred from different species.

699 Trinucleotide-context mutational spectra of signature SBSB, as inferred independently from

700 somatic mutations in crypts from four representative species (top to bottom): human, naked mole-

701 rat, rat, and rabbit (Methods). Signatures are shown in a human-genome-relative representation.

702 Cosine similarities between each signature and the human COSMIC SBS5 and SBS40 are

703 provided.

704
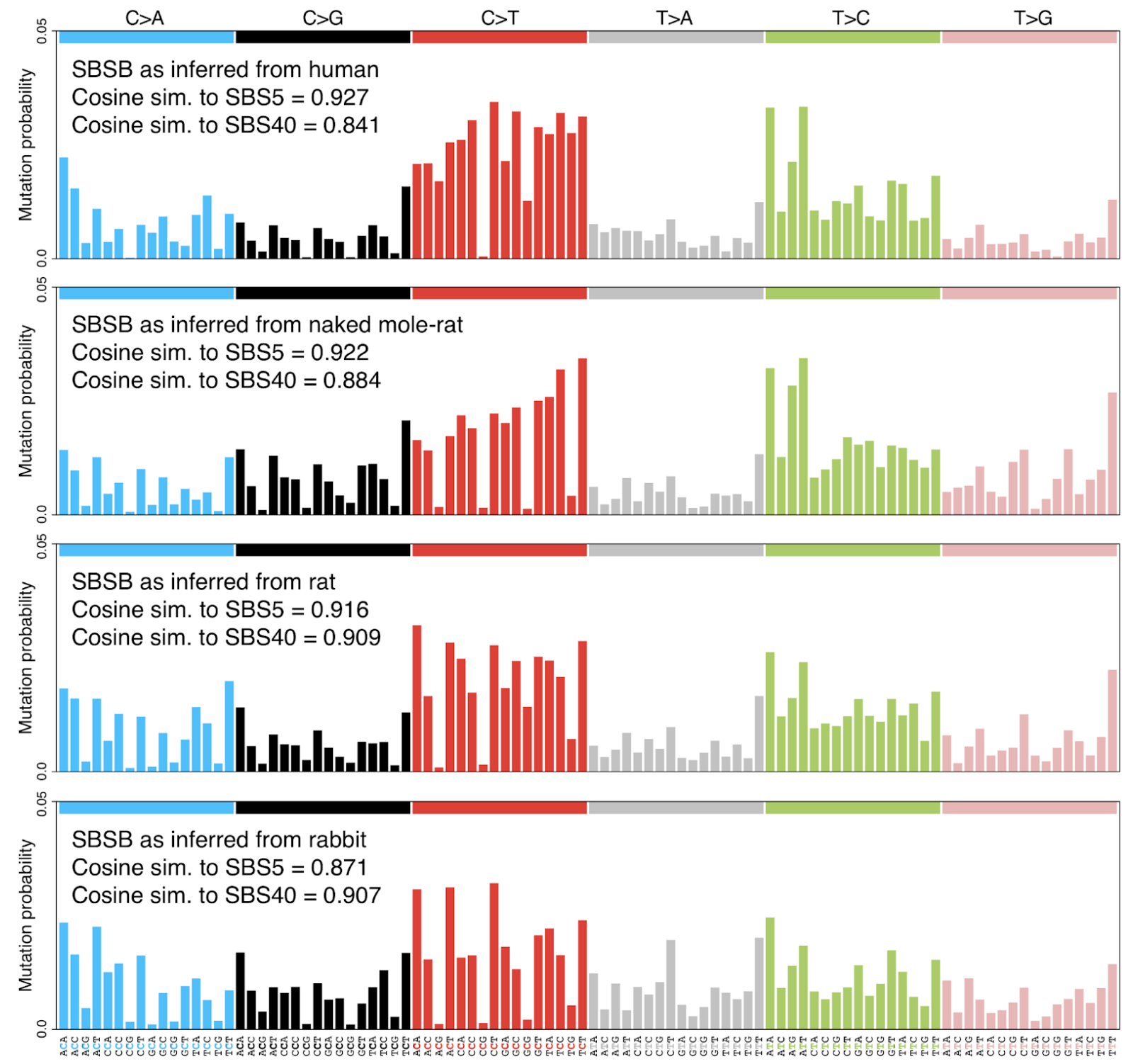
bioRxiv preprint doi: https://doi.org/10.1101/2021.08.19.456982; this version posted December 14, 2021. The copyright holder for this preprint (which was not certified by peer review) is the author/funder, who has granted bioRxiv a license to display the preprint in perpetuity. It is made available under aCC-BY-NC-ND 4.0 International license.

\section{Extended Data Figure 7. Mutational spectra of somatic indels in each species. Horizontal}

709 axis presents 83 insertion/deletion types, coloured by type and length ${ }^{36}$.

710
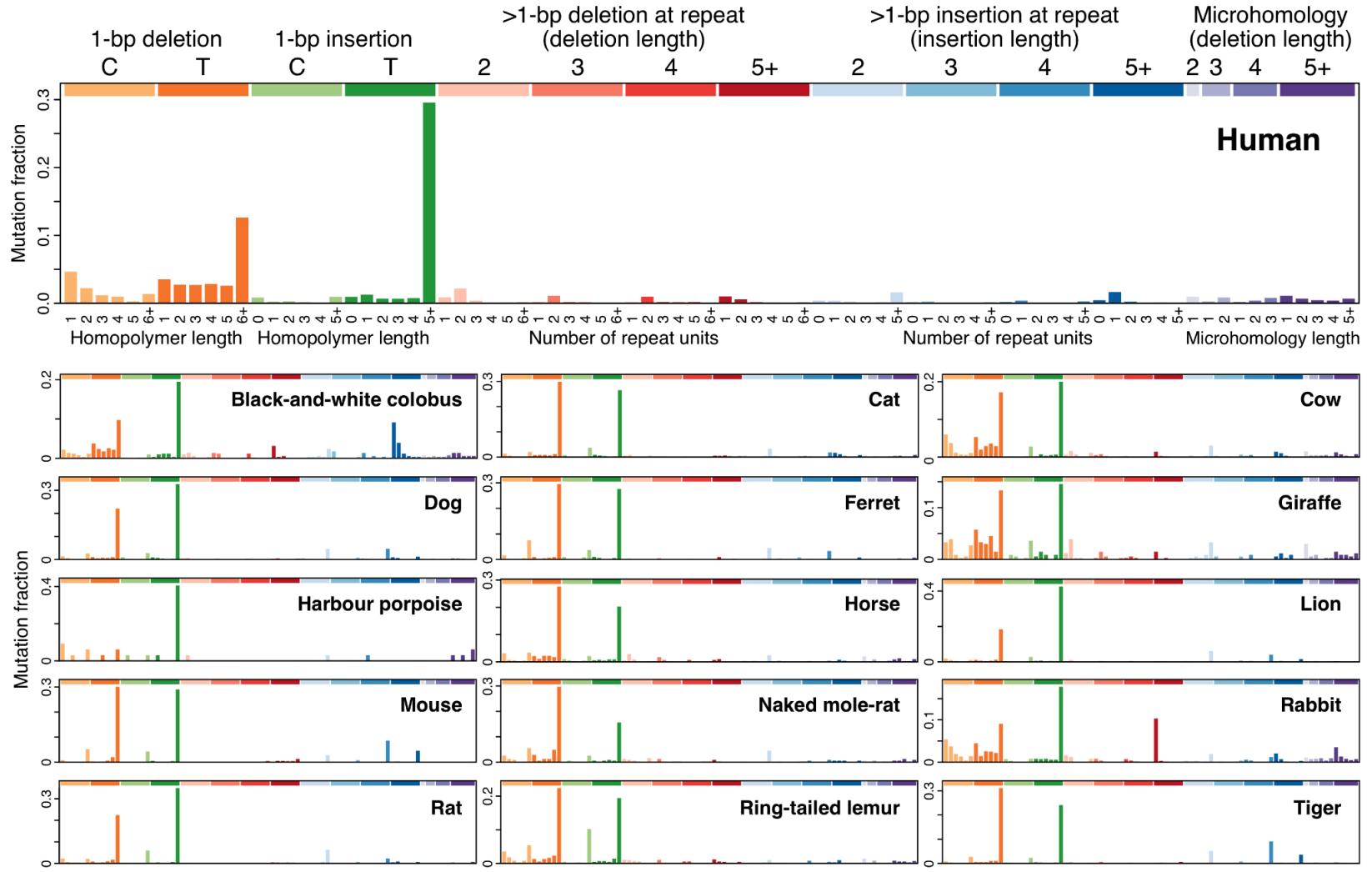


\section{Extended Data Figure 8. Colibactin exposure in non-human and human colorectal crypts.}

717 Exposures to mutational signatures SBS1, SBS5, SBS18, SBS34 and SBS88, as inferred via

718 expectation-maximisation, for 180 non-human crypts (top) and 445 human crypts sequenced in a

719 previous study $^{8}$. Asterisks indicate samples with statistically significant colibactin (SBS88)

720 exposure, based on a likelihood ratio test (Methods). BW, black-and-white; H, harbour; N, naked;

721 RT, ring-tailed.

722
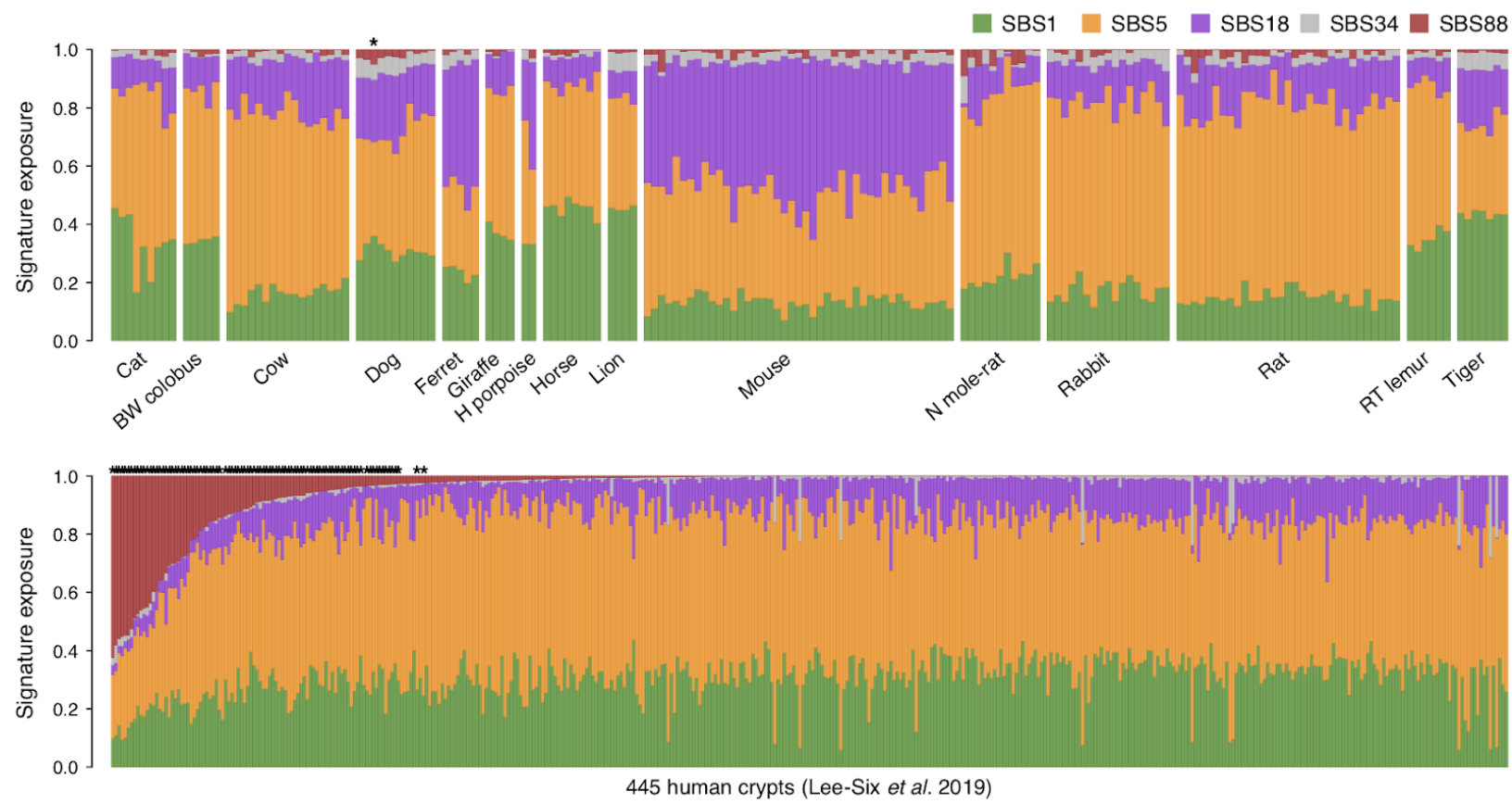
726 Extended Data Figure 9. Identified copy number changes. Somatic copy number changes in

727 cow (a, b), mouse (c) and human (d) colorectal crypts. For each case, chromosomes are presented

728 along the horizontal axis, and each point represents a 100-kb genomic bin. The top panel presents

729 the ratio between observed and expected sequencing coverage per bin; the middle panel shows

730 the median B-allele fraction (BAF) of heterozygous germline SNPs per bin; and the bottom panel

731 presents the inferred segments of total copy number (green) and allele-specific copy number

732 (red/blue). Regions of copy number change are highlighted in pink. The sparsity of BAF and

733 allele-specific copy number values in the mouse crypt (c) are due to the fact that mouse samples

734 generally had very low numbers of germline SNPs. 
bioRxiv preprint doi: https://doi.org/10.1101/2021.08 19.456982. this version posted December 14,2021 . The copyright holder for this preprint (which was not certified by peer review) is the author/funder, who has granted bioRxiv a license to display the preprint in perpetuity. It is made available under aCC-BY-NC-ND 4.0 International license.

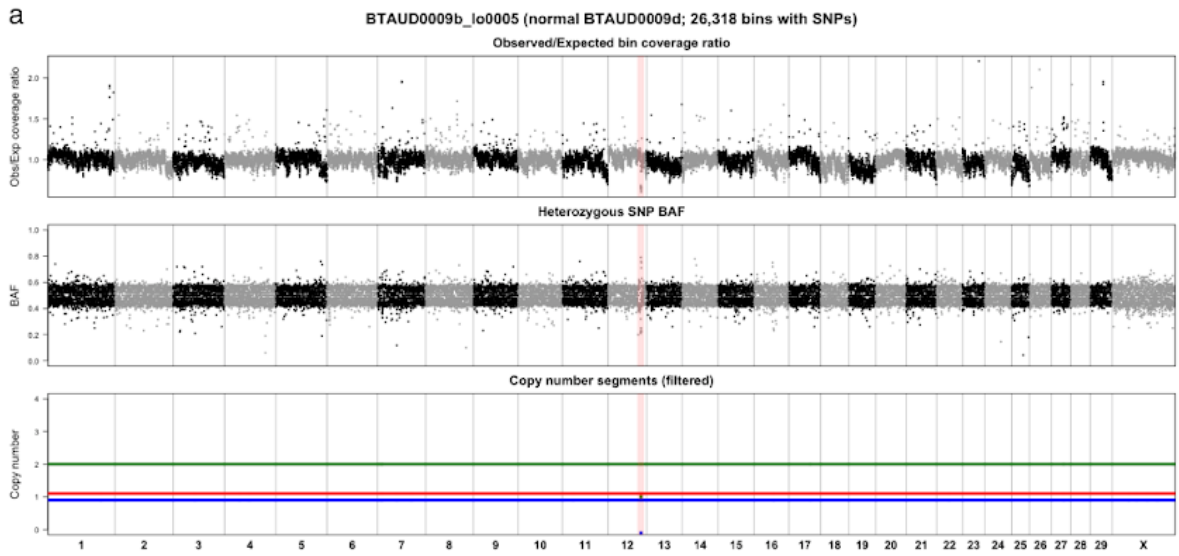

BTAUD0009b_100010 (normal BTAUD0009d; 26,286 bins with SNPs)
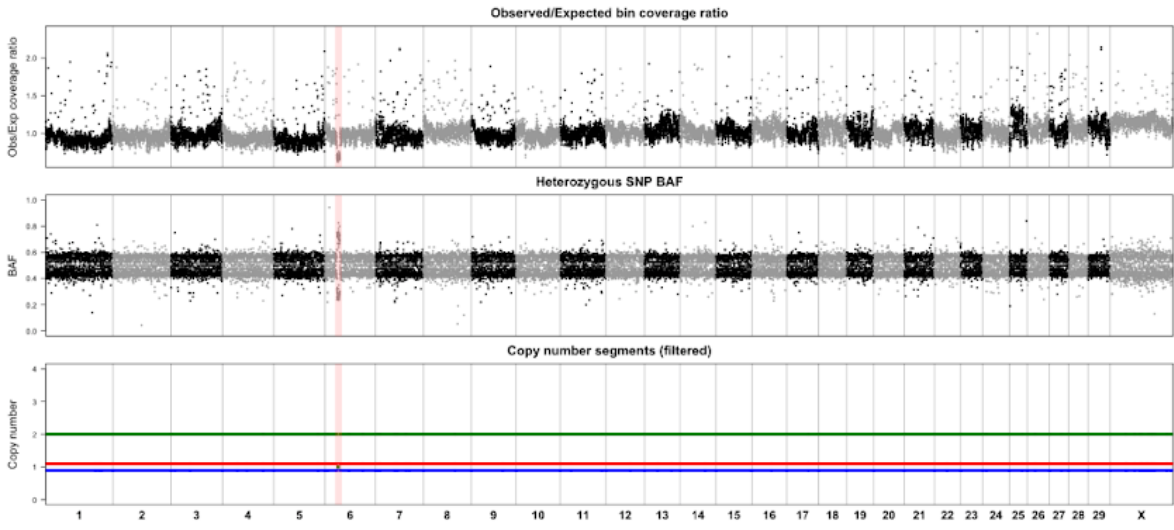

C
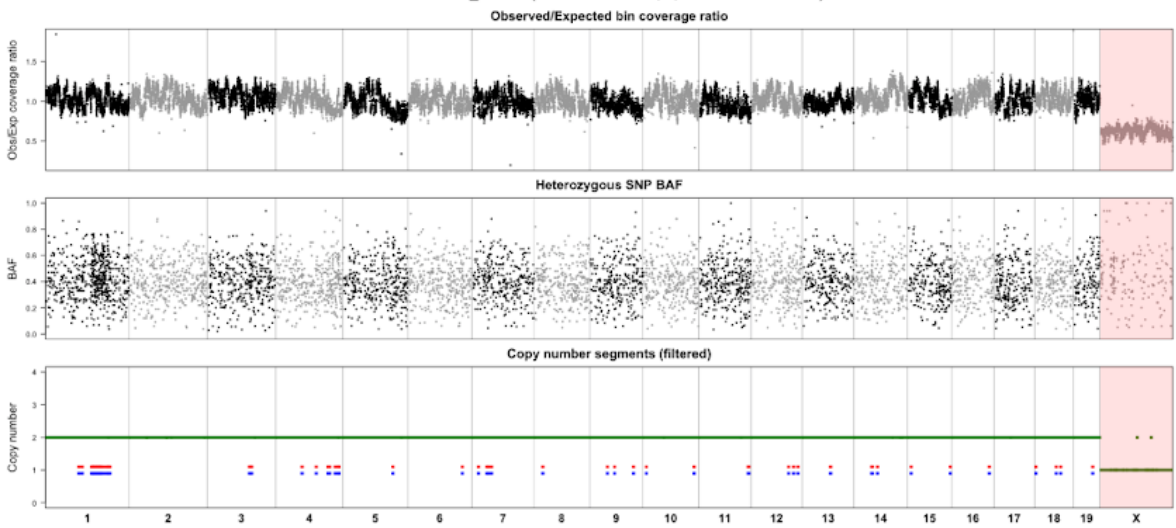

d $\quad$ PD36813ad8 (normal PD36813ad2; 27,966 bins with SNPs)
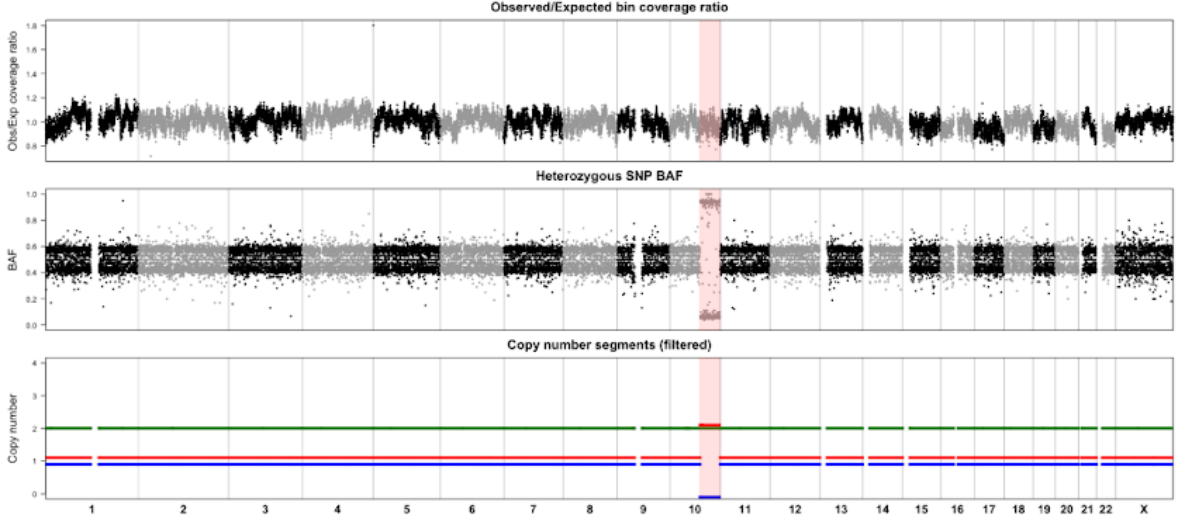
736 Extended Data Figure 10. Somatic dN/dS. Estimates of dN/dS for somatic missense and

737 truncating mutation in each of the species with available genome annotation. Dots and error bars

738 represent maximum likelihood estimates and 95\% confidence intervals, respectively. Note the

739 logarithmic scale of the vertical axis.

740

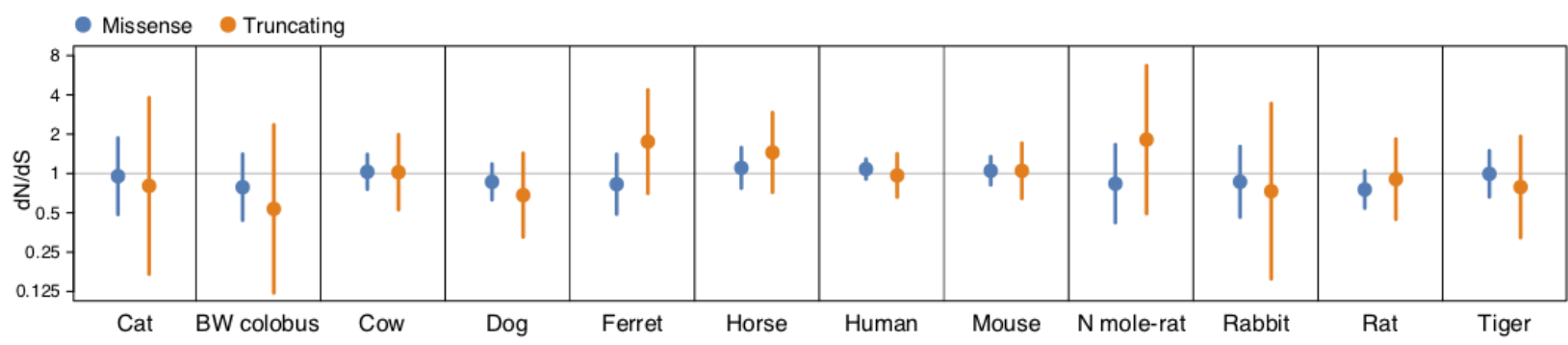




\section{Extended Data Figure 11. Kaplan-Meier curves of longevity in captivity. Kaplan Meier}

746 survival curves for each species using captive lifespan data from Species360 for non-human

747 species and census record data for humans (Methods). The red shaded areas represent 95\%

748 confidence intervals of the curve. A horizontal grey bar indicates the 80th percentile, which was

749 employed as a robust estimate of species lifespan.

750
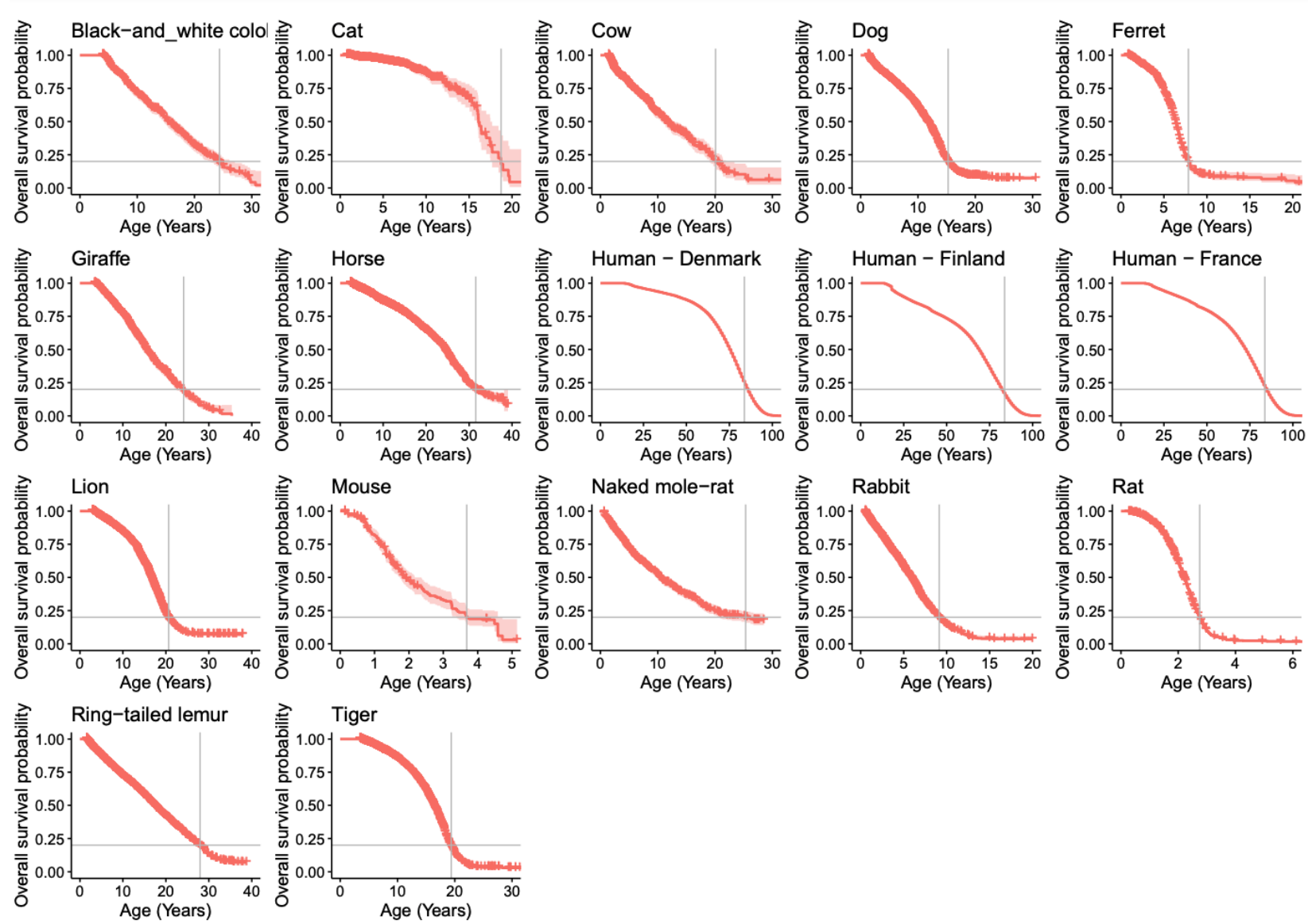

751 
753 Extended Data Figure 12. Associations between life-history variables and alternative

754 measures of somatic mutation rate. The figure presents the same analyses as Fig. 3d,e, but

755 employing somatic mutation rates per megabase (a), or per protein-coding exome (b), rather than

756 per genome (Methods). Leftmost panels show zero-intercept linear mixed-effects (LME)

757 regressions of somatic mutation rates on inverse lifespan (1/Lifespan), presented on the scale of

758 untransformed lifespan (horizontal axes). Vertical axes present mean mutation rate per species,

759 although mutation rates per crypt were used in the regressions. Darker shaded areas indicate 95\%

760 confidence interval (CI) of the regression lines; lighter shaded areas mark a two-fold deviation

761 from the regression line. Point estimate and 95\% CI of the regression slope coefficient $(k)$,

762 fraction of inter-species variance explained by the model (FVE), and range of end-of-lifespan

763 burden (ELB) are provided. Rightmost panels show comparisons of FVE values achieved by

764 free-intercept LME models using inverse lifespan and other life-history variables (alone or in

765 combination with inverse lifespan) as explanatory variables. BMR, basal metabolic rate; BW,

766 black-and-white; Mb, megabase; N, naked; RT, ring-tailed.
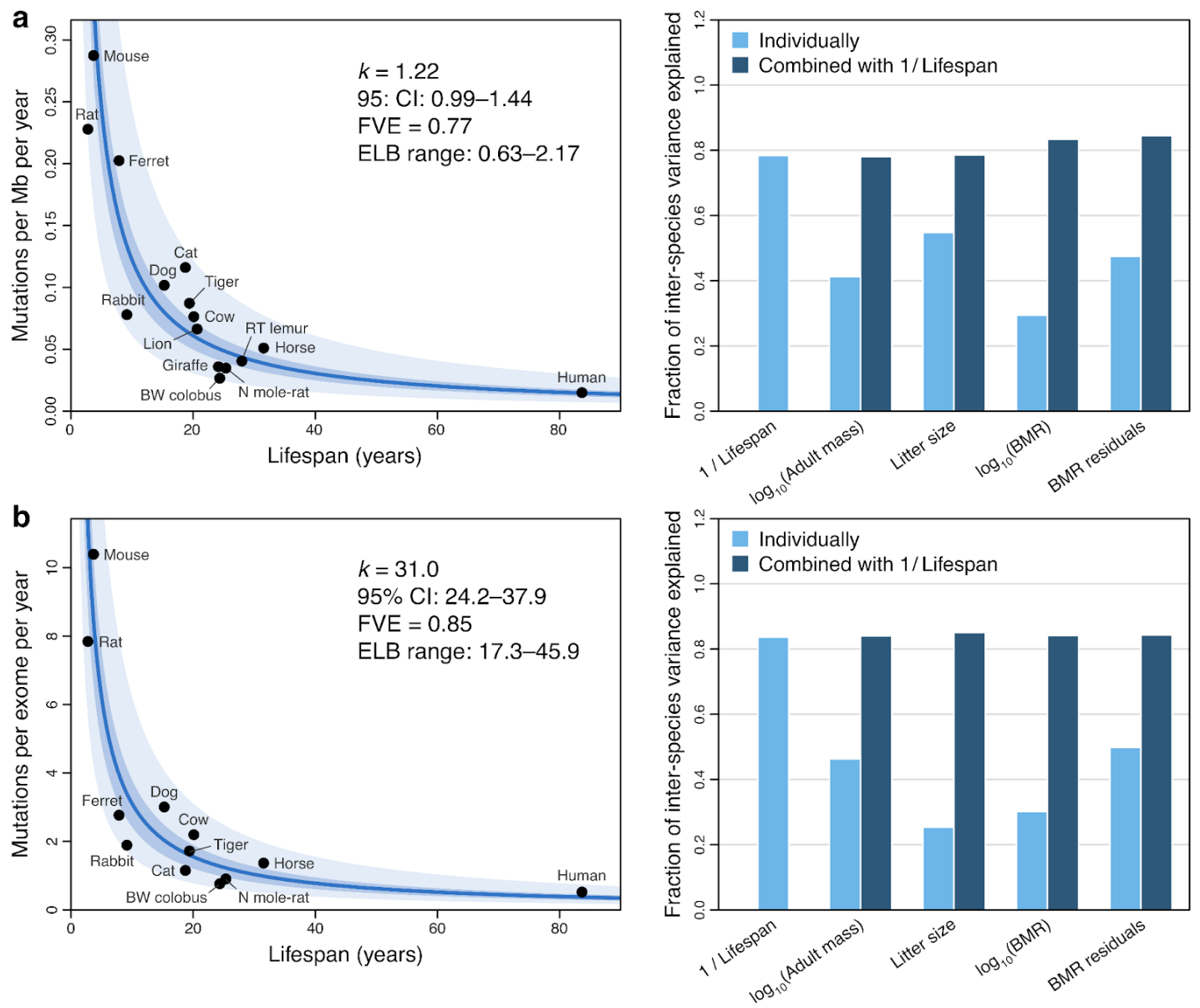
Extended Data Figure 13. Bootstrapped regression of somatic mutation rates on published

770 lifespan estimates. a, Bootstrapped regression of somatic substitution rates on the inverse of

771 lifespan (1/Lifespan), using a zero-intercept linear mixed-effects model (Methods). For each of

7725000 bootstrap samples, lifespan values per species were randomly selected from a set of

773 published maximum longevity estimates (Supplementary Table 6). The blue line indicates the

774 median regression slope $(k)$ across bootstrap samples, and the blue shaded area depicts the range

775 of estimates of $k$ across bootstrap samples. Black dots and error bars indicate the mean and range,

776 respectively, of published longevity estimates for each species. The median and range of both $k$

777 and the fraction of inter-species variance explained (FVE) are provided. b, Histogram of FVE

778 values across the 5000 bootstrap samples.

779
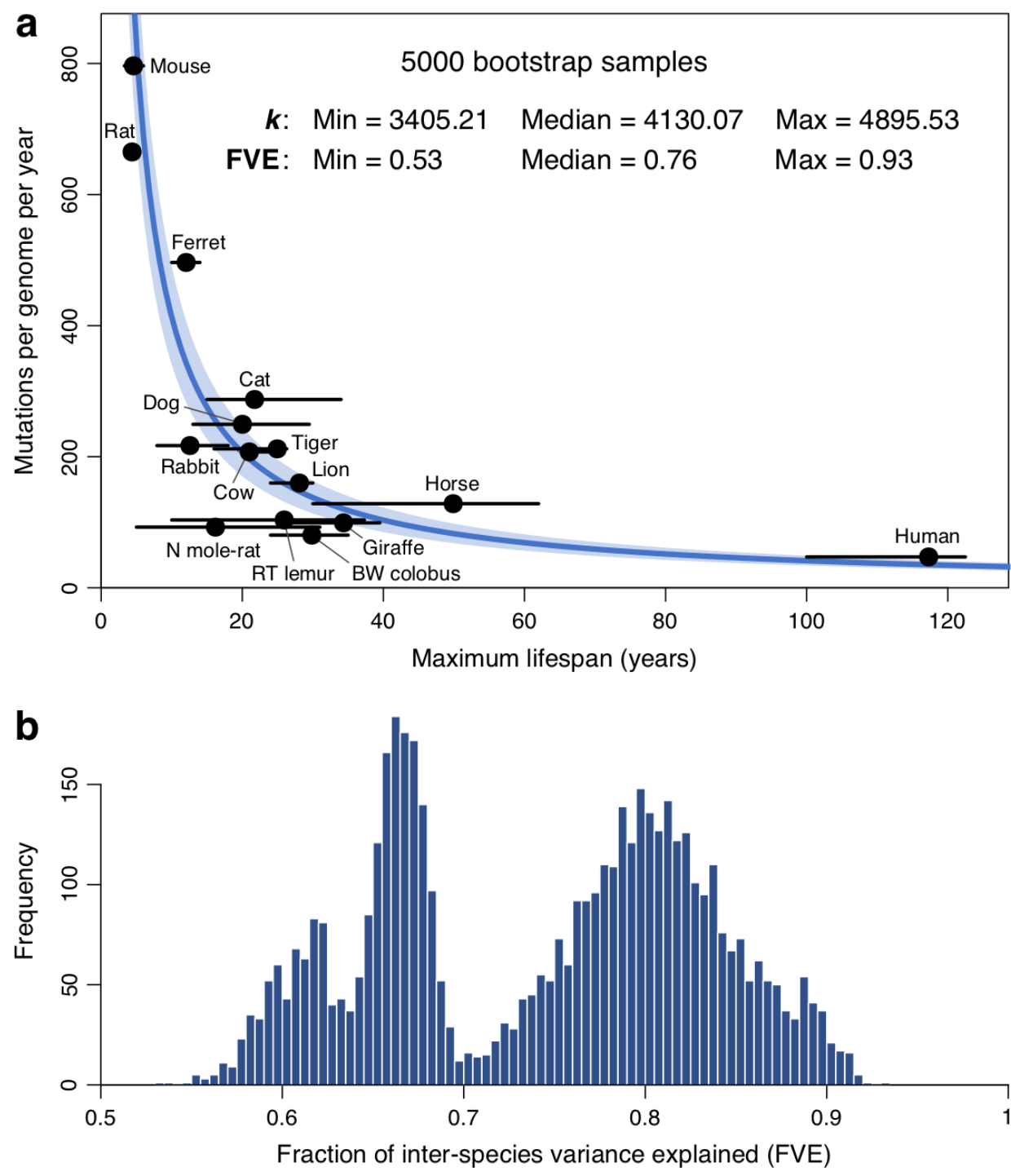


\section{Extended Data Figure 14. Comparison of regression models for somatic mutation rates. a,}

782 Constrained-intercept regression of somatic substitution rates on the inverse of lifespan

783 (1/Lifespan), using a linear mixed-effects (LME) model applied to the rates per crypt (left) and a

784 Bayesian hierarchical normal regression model applied to the mean rates per individual. For

785 simplicity, black dots present mean mutation rates per species. Darker shaded area indicates $95 \%$

786 confidence/credible interval (CI) of the regression line; lighter shaded area marks a two-fold

787 deviation from the regression line. Point estimates and 95\% CI of the regression slopes $(k)$ and

788 fraction of inter-species variance explained (FVE) are provided. b, Comparison of regression

789 lines for the of somatic substitution rates on 1/Lifespan (left; zero intercept) and log-transformed

790 adult mass (right; free intercept), using simple linear models (dark and light blue), phylogenetic

791 generalised least-squares models (orange and yellow), Bayesian hierarchical normal models

792 (green) and LME models (red). Point estimates of the regression coefficients for each model are

793 provided. c, Distributions of regression FVE under individual- and species-level bootstrapping.

794 For the LME models regressing somatic mutation rates on inverse lifespan (zero intercept; left)

795 and log-mass (free intercept), the curves present distributions of FVE from 10,000 bootstrap

796 replicates, obtained through random resampling of either individuals (blue) or species (orange)

797 (Methods). Vertical lines indicate the FVE values obtained using the entire data set. 
bioRxiv preprint doi: https://doi.org/10.1101/2021.08.19.456982; this version posted December 14, 2021. The copyright holder for this preprint (which was not certified by peer review) is the author/funder, who has granted bioRxiv a license to display the preprint in perpetuity. It is made available under aCC-BY-NC-ND 4.0 International license.
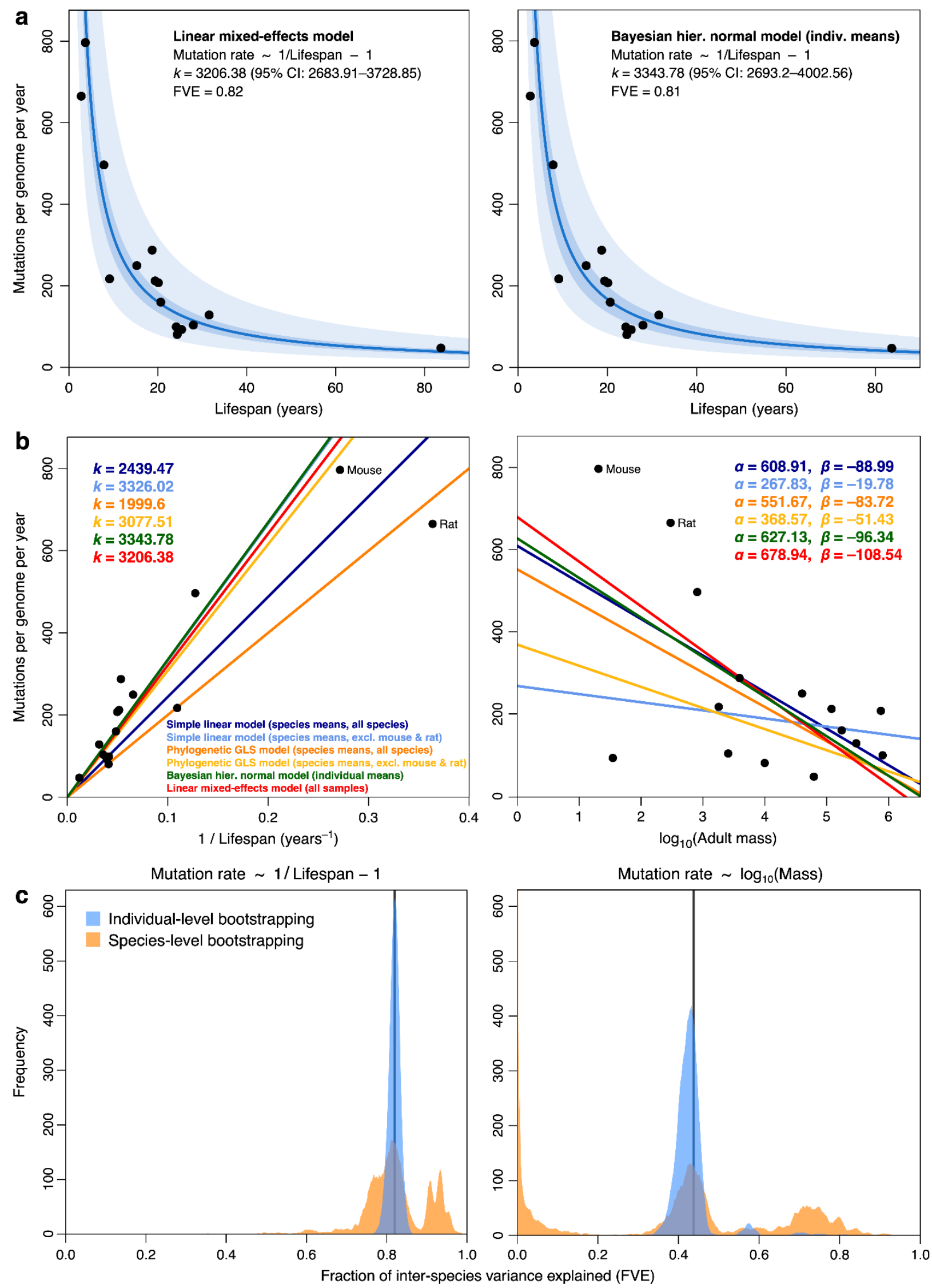
Extended Data Figure 15. mtDNA mutation burden and copy number. a, Total somatic

801 mtDNA mutations (substitutions and indels) called in each sample. b, Somatic mutation burden

802 per mitochondrial genome copy per sample. c, Estimated mtDNA copy number per sample.

803 Samples are arranged as in Fig. 1b, with samples from the same individual coloured in the same

804 shade of grey.

805
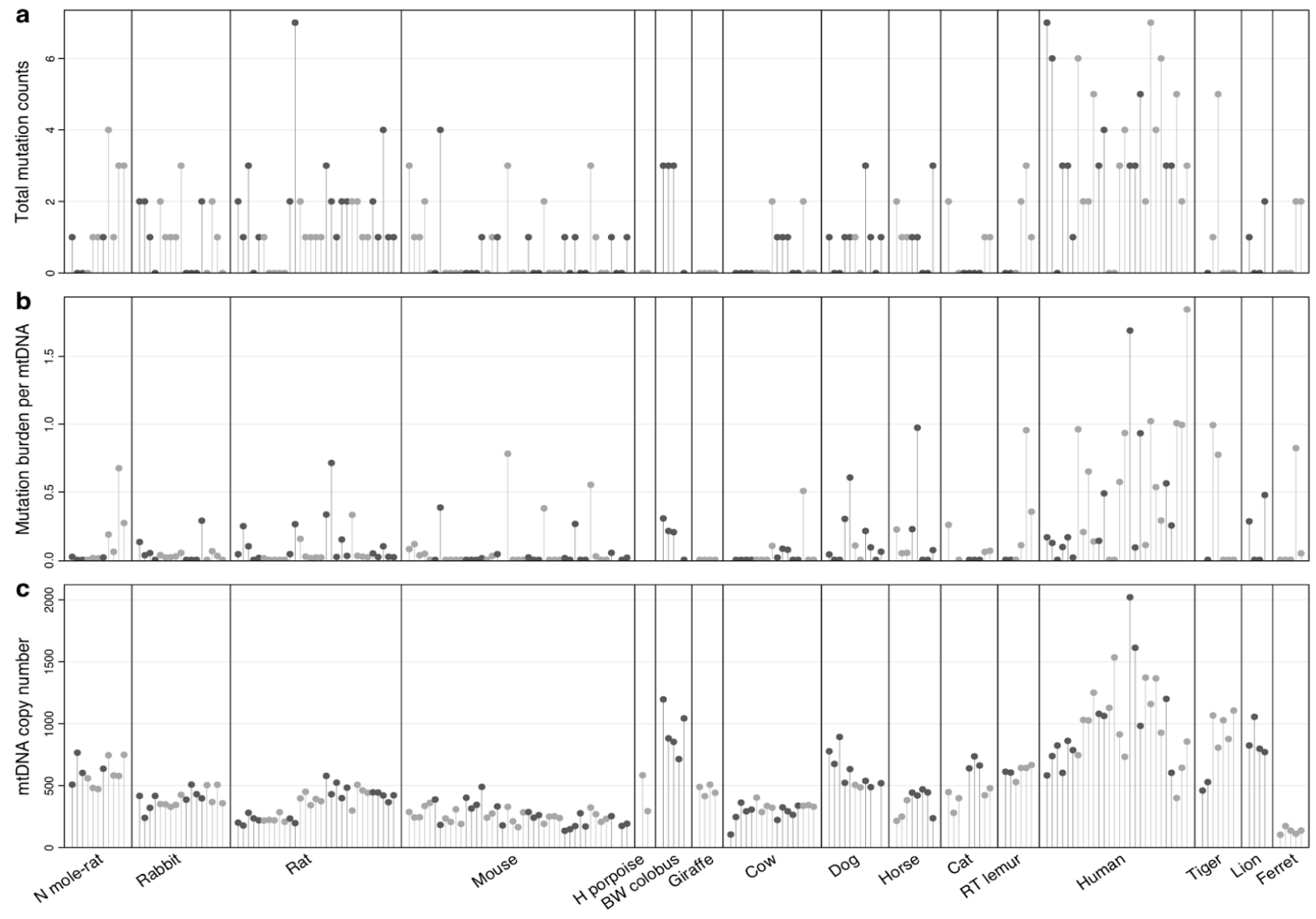
810 Horizontal axis presents 96 mutation types on a trinucleotide context, coloured by base

811 substitution type. Mutations on the upper and lower halves of the spectrum represent substitutions

812 in which the pyrimidine base is located in the heavy and light strands of mtDNA, respectively.

813
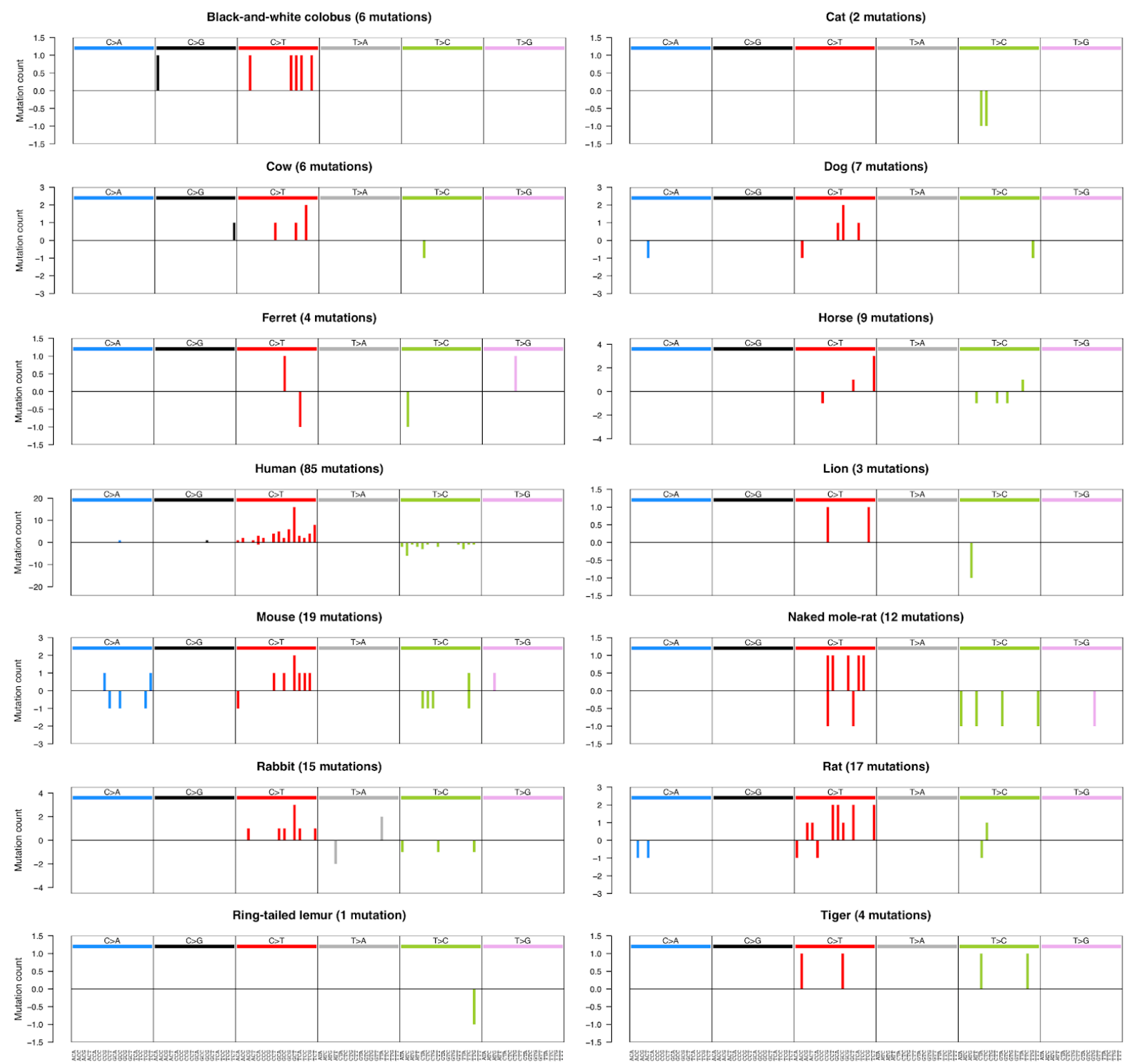
818 Extended Data Figure 17. Assessment of variant calling and filtering. a, Spectra of SBS calls

819 before (left) and after application of the final eight variant filters, across all giraffe samples. Note

820 that the set of 'unfiltered' variants (left) has gone through the three early filters named 'quality

821 flag filter', 'alignment quality filter' and 'hairpin filter' (Methods). b, Spectra of calls flagged as

822 artefactual by each of the final eight variant filters, across all giraffe samples. Sets of variants

823 flagged by different filters are not mutually exclusive. c, Venn diagram showing the number of

824 variant calls shared between two LCM sections from the same mouse colorectal crypt. d, Venn

825 diagram showing the numbers of variant calls shared between five different colorectal crypts

826 from the same mouse.

827
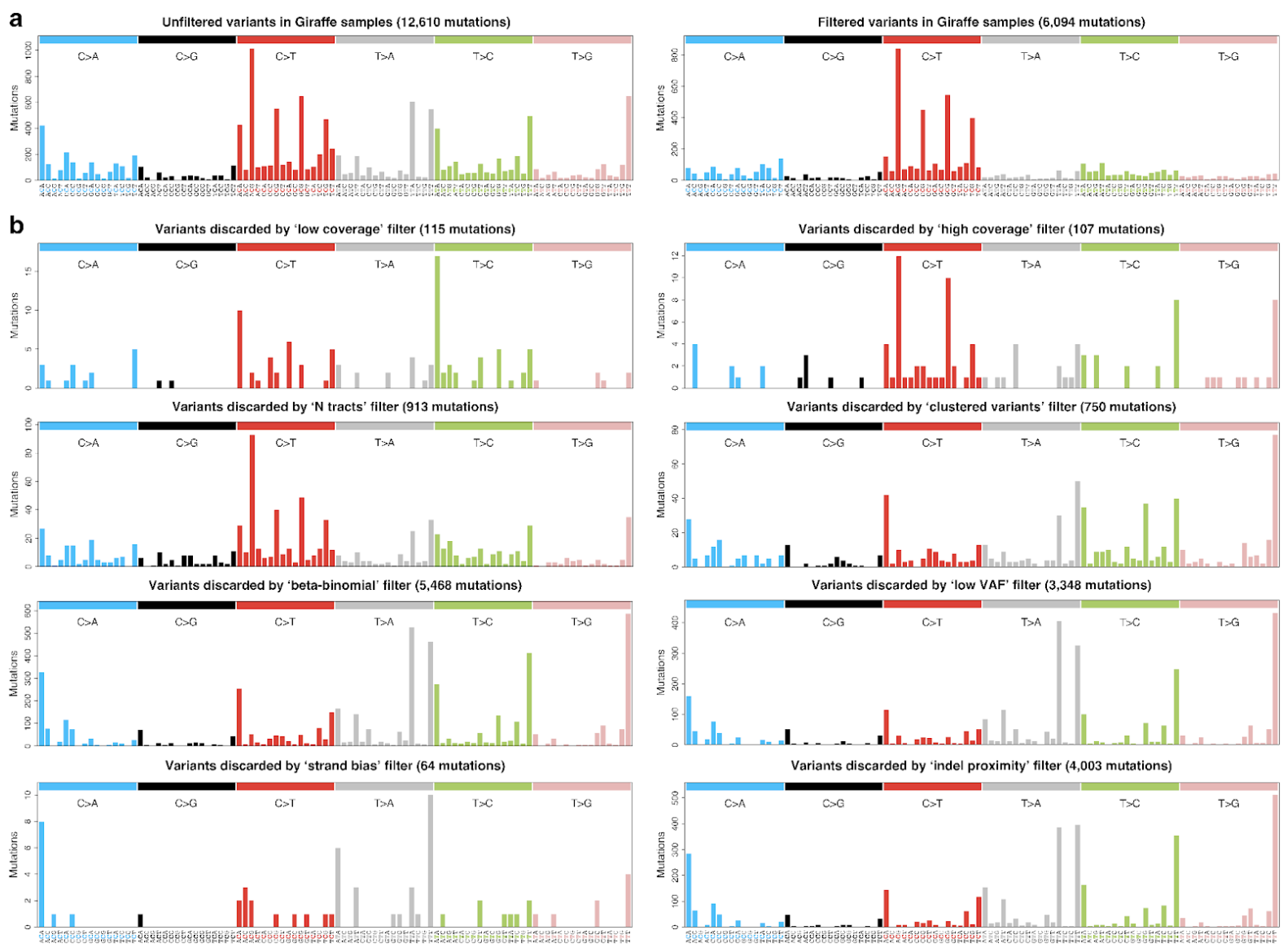

C
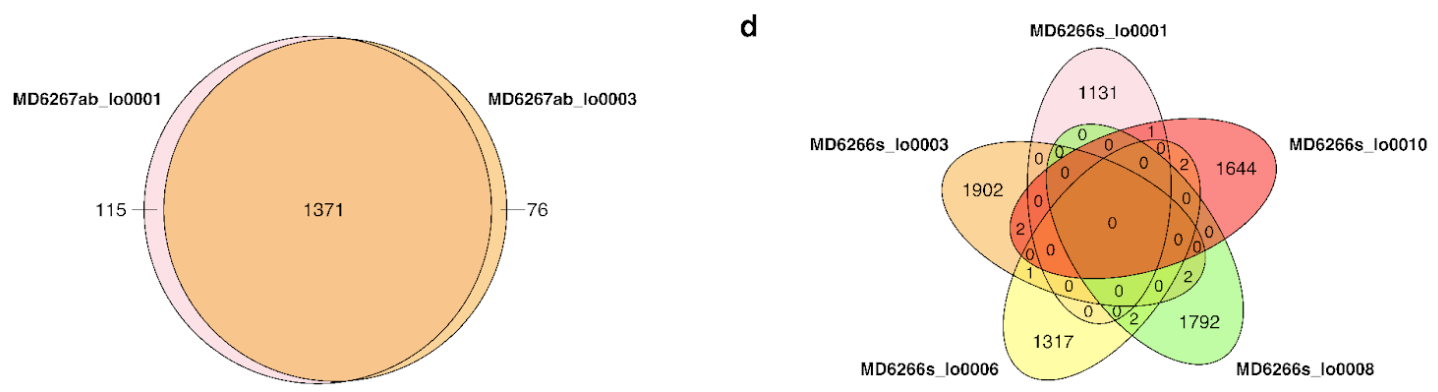
829 Extended Data Figure 18. Mutational signatures and exposures inferred de novo. a,

830 Mutational signatures inferred de novo from the species mutational spectra shown in Fig. 2 a.

831 Signatures are shown in a human-genome-relative representation. SBSA is the de novo

832 equivalent of COSMIC signature SBS1 (Fig. 2b). b, Exposure of each sample to each of the

833 mutational signatures shown in a. Samples are arranged horizontally as in Fig. 1b. c, Regression

834 of signature-specific mutation burdens on individual age for human, mouse and naked mole-rat

835 samples. Regression was performed using mean mutation burden per individual. Shaded areas

836 indicate $95 \%$ confidence intervals of the regression line. BW, black-and-white; H, harbour; $\mathrm{N}$,

837 naked; RT, ring-tailed.

838
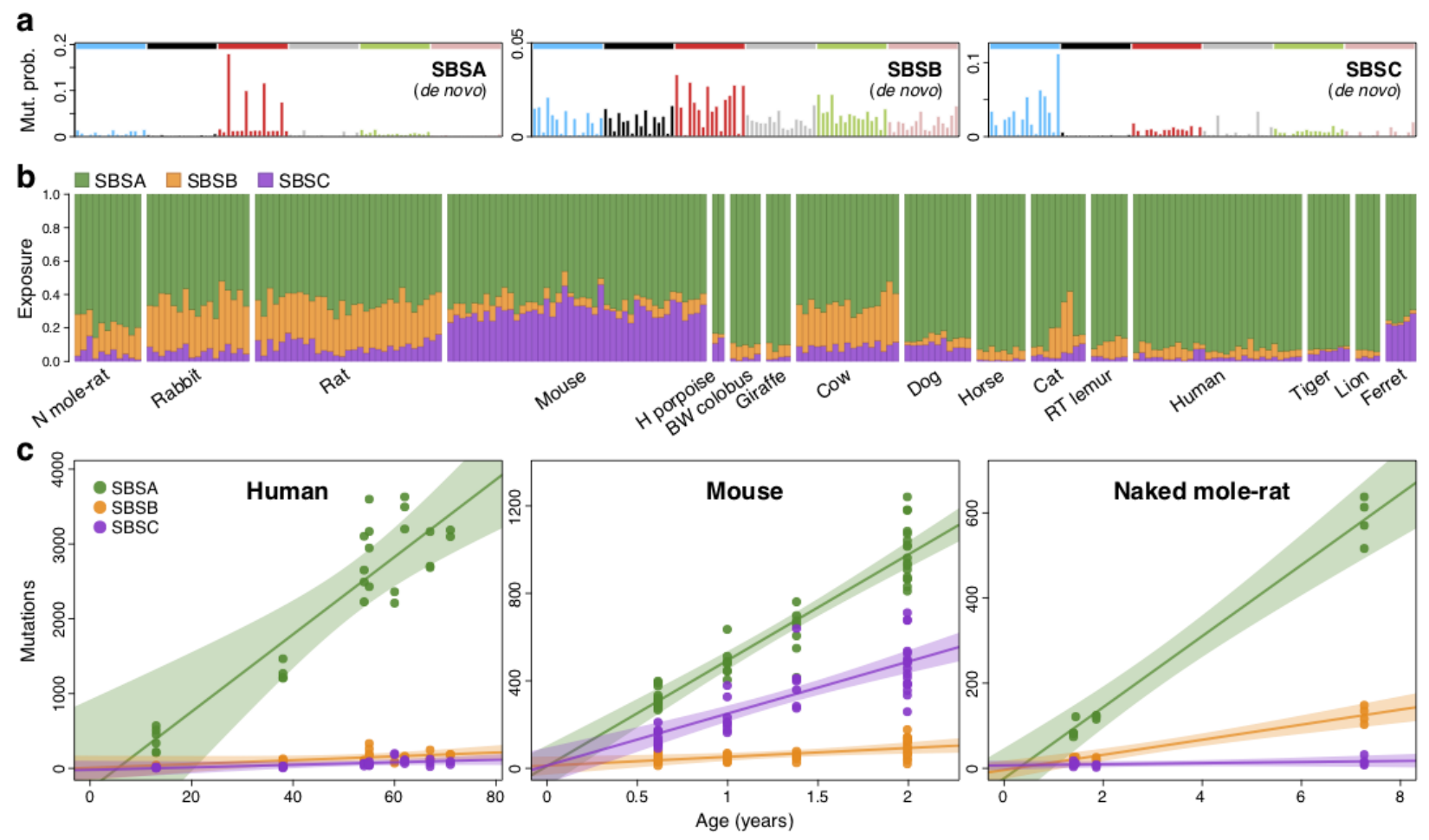
845 Supplementary Table 1. Species information. For each of the (sub)species in the study, the

846 table provides: common name, scientific name, number of individuals in the study, number of

847 colorectal crypts sequenced, range of individual ages, and source institution.

849 Supplementary Table 2. Sample information. For each colorectal crypt sample in the study,

850 the table provides: sample ID, individual ID, species name, matched normal sample ID, matched

851 normal sample type, and median sequencing depth.

854 each species in the study (except harbour porpoise), the table provides the mean observed values

855 of the rate of somatic substitutions per genome per year, and point estimates and 95\% confidence

856 intervals for simple linear regression of mean substitution burdens per individual on individual

857 ages. The estimated regression slopes correspond to the estimated mutation rate per year for each

858 species. Estimates are provided for constrained-intercept linear models applied to all species, and

859 for free-intercept linear models applied to the eight species with at least three individuals.

Supplementary Table 4. Somatic mutation burdens, rates and signature exposures. For each colorectal crypt sample in the study, the table provides: sample ID, individual ID, species name, individual age, total genome size, coding genome size, analysable genome size, analysable mtDNA size, mutational signature exposures (SBS1, SBSB, SBSC); somatic mutation burdens per genome for single-base substitutions, indels, signature-specific substitutions (SBS1, SBSB, SBSC), and mtDNA mutations; and somatic mutation rates per genome per year for single-base substitutions, indels, signature-specific substitutions (SBS1, SBSB, SBSC), and mtDNA

868 mutations.

870 Supplementary Table 5. Reference genome information. For each species in the study, the table provides: reference genome version used, reference mtDNA sequence used, Ensembl

872 genome annotation version used (where applicable), reference genome file source, reference 873 mtDNA file source, reference genome file URL, reference mtDNA file URL. 
875 Supplementary Table 6. Life history data. For each species in the study (except harbour

876 porpoise), the table provides: adult mass (g), basal metabolic rate (W), litter/clutch size,

877 maximum longevity (years); and maximum likelihood estimate and 95\% confidence limits for the

878 estimated $80 \%$ lifespan, together with the corresponding sample size. The source of each estimate

879 is given in brackets.

880

881 Supplementary Table 7. Number of cell divisions per lifespan. For mouse, rat and human, the

882 table provides: estimated rate of colorectal cell division (hours), estimated lifespan (years),

883 estimated number of cell division at the end of lifespan, estimated number of mutations per cell

884 division (obtained using our mutation rate estimates), and the reference for the cell division rate.

885 For humans, two estimates of cell division rate are included.

886 